\title{
31. MAJOR AND TRACE ELEMENT GEOCHEMISTRY OF OLIGOCENE TO QUATERNARY VOLCANICLASTIC SANDS AND SANDSTONES FROM THE IZU-BONIN ARC ${ }^{1}$
}

\author{
Richard N. Hiscott ${ }^{2}$ and James B. Gill ${ }^{3}$
}

\begin{abstract}
During Ocean Drilling Program Leg 126, six sites were cored in a young backarc rift basin and its flanks (rift onset 1.1-3.56 Ma) and in the forearc basin of the Izu-Bonin Arc. In the backarc area, strata are younger than about 4.5 Ma, whereas in the forearc, ages are about $0-31 \mathrm{Ma}$ in sections punctuated by important Miocene unconformities. Bulk chemical analyses of volcaniclastic turbidite sands and sandstones, derived directly from the arc, were obtained from 271 atomic absorption analyses (major elements), $253 \mathrm{XRF}$ analyses (trace elements) and 16 ICP-MS analyses (trace and rare-earth elements). Of the 271 samples, 78 come from the backarc area and the remainder from the forearc.

The sands and sandstones reflect the igneous compositions of their sources. Most are formed of materials derived from subalkaline, low- $\mathrm{K}$ andesites, and dacites, although compositions range from basalt to rhyolite. Basic and acid andesites are predominant in Oligocene rocks; in contrast, Pliocene-Pleistocene sediments were derived from acid andesitic to rhyolitic sources. The oldest sandstones, estimated to have an age of about $31 \mathrm{Ma}$, were derived from an arc tholeiitic, not boninitic, source. The 26-31 Ma sandstones furthest to the north, at Sites 787 and 792, have higher relative concentrations of $\mathrm{Ti}, \mathrm{Zr}$, and $\mathrm{Y}$ than do those at southern Site 793. Data from younger samples indicate that, for more than 30 m.y., the average composition of volcaniclastic sediments and volcanism near Aoga Shima was more basic than to the south, near Sumisu Jima.

Using the sandstones as igneous proxies, we conclude that magmas erupted along the arc have become more depleted in light-rare-earth elements (LREE) with time. There was a major change in rare-earth-element (REE) concentrations in the late Oligocene, from essentially flat patterns (normalized $\mathrm{La} / \mathrm{Yb}$ about 1-1.5) to LREE-depleted patterns (normalized $\mathrm{La} / \mathrm{Yb}$ about 0.5 ). At the same time, $\mathrm{Zr} / \mathrm{Y}$ ratios decreased from about $2-4$ to about 1.5-2.5. These changes may reflect a shift in provenance, or changes in the composition of the mantle wedge beneath the arc.

In the backarc area, lithic clasts and glass shards of rift-facies basalt are present in sediments as old as $2.35-3.15 \mathrm{Ma}$. Two samples of mafic sand from the backarc basin have flat REE patterns (normalized $\mathrm{La} / \mathrm{Yb}$ about 1.0), like some of the $<1$-Ma rift lavas and unlike pre-rift sand and sandstone samples. These possibly represent the local effects of sedimentary mixing of detritus from arc and backarc eruptions because no evidence from the arc itself exists to suggest a recent change in the REE content of magmas.
\end{abstract}

\section{INTRODUCTION}

Sediments and sedimentary rocks were recovered from six Ocean Drilling Program (ODP) Leg 126 sites around the Izu-Bonin Arc: two in the backarc Sumisu Rift, one on the eastern uplifted flank of the rift, and three in the forearc basin (Fig. 1). Except for a generally small biogenic fraction, the detritus in the sediments and sedimentary rocks was derived entirely from volcanoes in the vicinity of this intraoceanic arc (Marsaglia, this volume). Some beds of sand-sized material recovered in cores appear to be primary waterlain ashes, but a far greater number are turbidites that contain mixed detritus resedimented from aprons around the volcanic centers into deep water. We follow Cas and Wright (1987) and apply sedimentary names to these resedimented volcaniclastics. The geochemistry of first-cycle detritus from intraoceanic arcs is poorly known (Taylor and McLennan, 1985; McLennan et al., 1990); the Leg 126 material provides an opportunity to accumulate important baseline data from this setting, as well as to track the geochemical evolution of the Izu-Bonin Arc itself.

The ages of the recovered volcaniclastic sediments range from late early Oligocene (about $31 \mathrm{Ma}$ ) to late Quaternary, although a few Eocene foraminifers from the basal strata at Site 792 (Herman, this volume), below an unconformity, may indicate locally older deposits. Throughout this time, the eastern margin of the Philippine Sea Plate was the site of subduction, producing a series of intraoceanic island

\footnotetext{
${ }^{1}$ Taylor, B., Fujioka, K., et al., 1992. Proc. ODP, Sci. Results, 126: College Station, TX (Ocean Drilling Program).

${ }^{2}$ Earth Sciences Department and Centre for Earth Resources Research, Memorial University of Newfoundland, St. John's, Newfoundland A1B 3X5, Canada.

${ }^{3}$ Department of Earth Sciences, University of California, Santa Cruz, CA 95064 U.S.A.
}

arcs (Honza and Tamaki, 1985). Little is known directly about the chemical composition of these volcanoes; the modern arc volcanoes are very young (Quaternary) and their substrate is unknown. To the east, along the modern outer arc high, Eocene volcanic rocks are exposed in the Bonin Islands (Kuroda and Shiraki, 1975; Tsunakawa, 1983; Matsuda, 1985) and at Leg 125 sites (Fryer, Pearce, et al., 1990); some of these Eocene rocks are type boninites, whereas others are arc tholeiites and calc-alkaline rocks. To the west, part of the early arc forms the Palau-Kyushu Ridge; this ridge was separated from the present arc when the Shikoku Basin formed from 17 to $25 \mathrm{Ma}$ (Kobayashi and Nakada, 1979). The Palau-Kyushu Ridge is underlain by middle Oligocene interbedded tuffs and chalks, and a volcanic basement consisting mostly of breccias and lavas of arc-tholeiitic composition. These are mostly basalts with $\mathrm{Zr} / \mathrm{Y}=1.4-2.5,(\mathrm{Ce} / \mathrm{Y})_{\mathrm{N}}=1-2$ (where subscript $\mathrm{N}$, throughout the paper, refers to values normalized to primitive mantle compositions of Table 1), flat REE patterns, $\mathrm{Ba}=50 \mathrm{ppm}$, and $\mathrm{Pb}=5 \mathrm{ppm}$ (Mattey et al., 1981; Migdisov, 1981; Scott, 1981, 1983).

To investigate the temporal variation of magma compositions in the Izu-Bonin Arc, 271 samples of volcaniclastic sand and sandstone were collected from cores at the six Leg 126 sites. Samples were split into three equal parts: one part for major-component petrography (Marsaglia, this volume), one part for electron microprobe analysis of mafic minerals and glass (Fujioka and Saito, this volume), and one part for bulk chemical analysis (this paper). The age distribution of the samples, based on sediment accumulation rates for the sites (Taylor, Fujioka, et al., 1990), is given in Figure 2. Because of low accumulation rates in the Miocene and the fine grain size of Miocene rocks, this time interval yielded a small number of samples. Several time gaps are present in the sample coverage (Fig. 2), but these are thought to be small enough that a general interpretation of arc evolution from the middle Oligocene to the present is not impeded. 


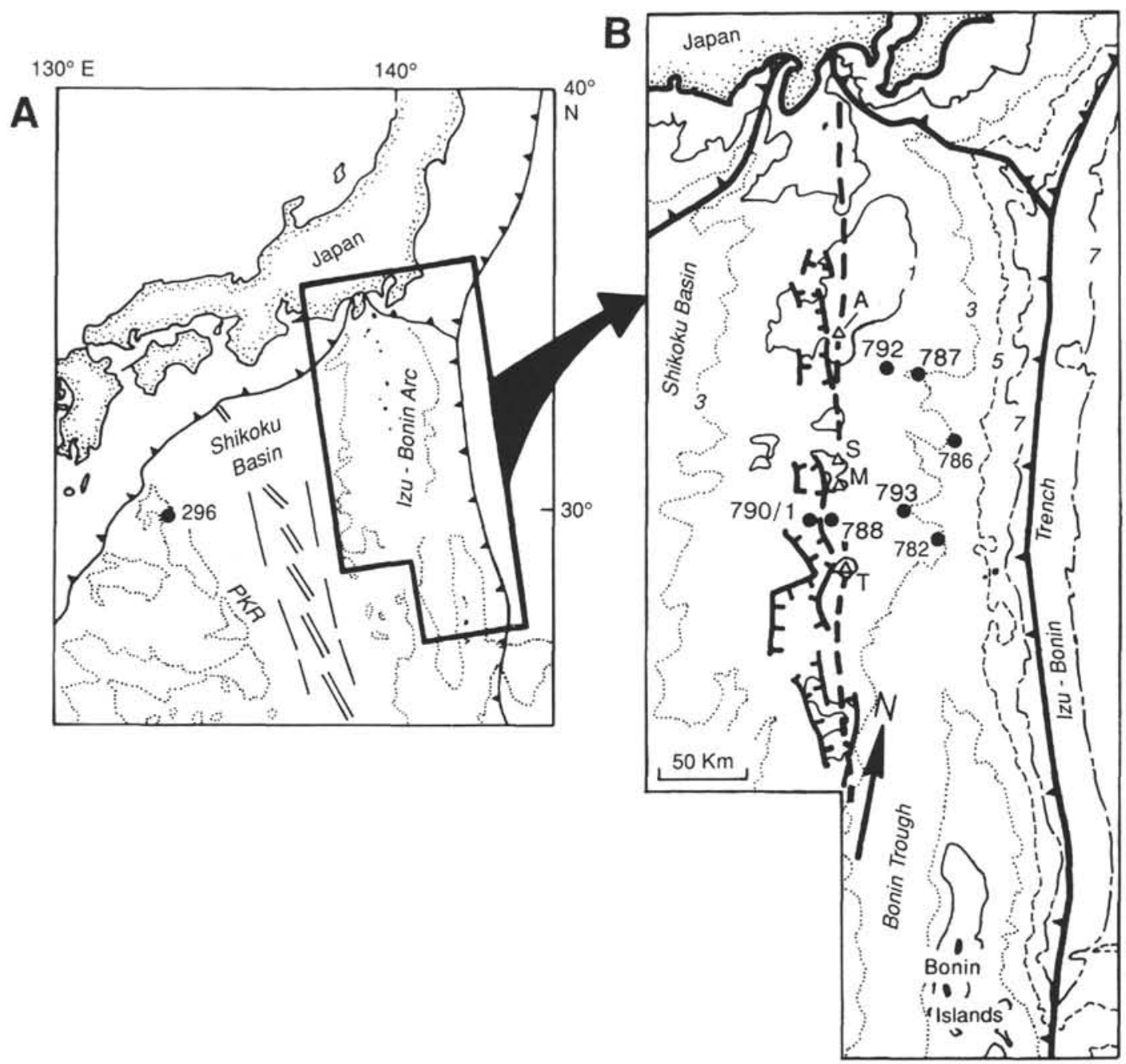

Figure 1. Location of drill sites. A. Position of the Izu-Bonin Arc at the eastern edge of the Philippine Sea Plate. DSDP Site 296 on the Palau-Kyushu Ridge (PKR) and the location of Figure 1B are indicated. The dotted lines mark 3-km water depth, the barbed lines are trenches, and the double lines indicate the extinct spreading center in the Shikoku Basin. B. Bathymetry, in thousands of meters, of the northern part of the Izu-Bonin Arc, from Honza and Tamaki (1985). Also indicated are the locations of Leg 126 drill sites in the backarc Sumisu Rift (Sites 790 and 791), the uplifted eastern flank of the rift (Site 788), and the Izu-Bonin forearc basin (Sites 787, 792, and 793). Leg 125 Sites 782 and 786 are also shown. Trenches are indicated by barbed lines and backarc basins by hachured lines. The position of the modern volcanic front is marked by a heavy dashed line that passes, among others, through arc volcanoes Aoga Shima (A), Sumisu Jima (S), Minami Sumisu Jima (M), and Tori Shima (T).

Detritus from arc volcanoes may not accurately reflect the sourcerock composition because of weathering in the source area, dilution by other components, hydraulic sorting during transport by currents, and postdepositional diagenesis. For Quaternary samples that have not undergone significant diagenesis, most grains are minimally altered glass and crystals (Marsaglia, this volume). Rapid transport into the deep basins around the arc prevented significant preburial chemical alteration of the detritus for these young sediments as well as for older turbidite sandstones (see below).

Hydraulic sorting operates on density and size differences between particles. The turbidite sands and sandstones that were sampled are generally very poorly sorted; samples were routinely taken from the more poorly sorted $\mathrm{a}$ and $\mathrm{b}$ divisions of turbidites (Bouma, 1962), or from thick massive sandstone units. For turbidites where recycling of grains is important, McLennan et al. (1990) demonstrate that there may be compositional contrasts with interbedded muds that result from a dependence of mineralogy on particle size during transport. We have no evidence for important recycling of components in this intraoceanic setting, and therefore we think that the effect of hydraulic sorting, as either mixing or unmixing of primary volcanic components, is minor.

Diagenesis of pre-Quaternary sandstones is locally severe, with primary glass and mafic crystals altered to zeolites and clay minerals (Marsaglia and Tazaki, this volume; Tazaki and Fyfe, this volume). Nevertheless, very high formation factors (Egeberg and the Leg 126 Shipboard Scientific Party, 1990), particularly in the Oligocene turbidite succession of the forearc basin, suggest that pore-water mobility has been small and that few reaction products have been able to escape from the rock. Instead, the diagenetic system in this part of the section was effectively closed, and reaction products have remained in the rock through incorporation into new minerals. Even the manganese staining in the upper section at Site 788 (Taylor, Fujioka, et al., 1990) appears to have changed nothing other than Mn contents (see Table 2).

We assume for the reasons outlined above that, for many elements, the bulk chemical composition of the sand and sandstone samples can be used as a crude proxy for the contemporaneous average composition of arc magmas. The word "average" must 


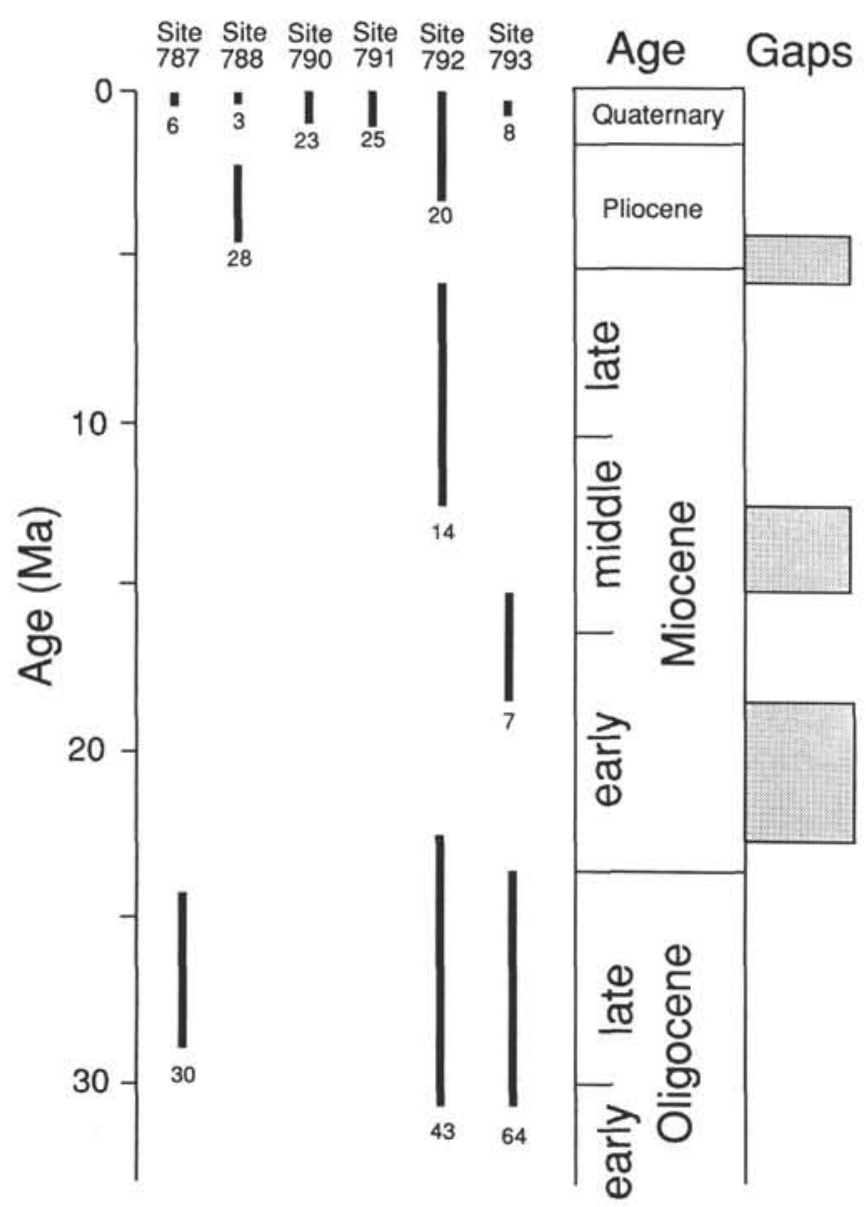

Figure 2. Number of samples and their age ranges at Leg 126 drill sites. Note the gaps in coverage in the Miocene and Pliocene caused by unconformities and lack of recovery. Miocene sampling is limited because of the low sedimentation rates and low sandstone abundance in the succession. The exact ages of samples older than $29 \mathrm{Ma}$ at Site 792 are not known, and they may be as old as $34 \mathrm{Ma}$ (Taylor, Fujioka, et al., 1990).

be emphasized, because bulk chemical data alone cannot be used to evaluate the extent of within-sample mixing of detritus with different compositions or even different ages. Petrographic examination of the youngest samples used in this paper reveals that they are generally mixtures of pale-to-black glass (Marsaglia, this volume). Electron microprobe analyses of individual glass grains in $0-1 \mathrm{Ma}$ ash beds, texturally similar to our sand and sandstone samples, indicates broad ranges in $\mathrm{SiO}_{2}$ content, from $56 \%$ to $80 \%$ (Rodolfo et al., this volume). Thus, even single eruptions may have produced compositionally variable ejecta. Turbidites derived from further mixing of primary material, including scattered hard-rock clasts eroded from the volcanoes, represent a further averaging of arc magma chemistry. Consequently, a sandstone of dacitic composition may consist primarily of andesitic and rhyolitic glass shards plus associated crystals, in any proportion.

\section{METHODS}

Approximately half of the samples were unconsolidated, and half lithified. The former were dried and split into three parts by K. Marsaglia, and the latter were cut into three parts by R. N. Hiscott using a rock saw. All saw marks were removed before further treatment. One of the splits from each rock sample was broken into chips with a maximum size of about $1 \mathrm{~mm}$, and then all samples were desalted by rinsing them three times in distilled water. After drying, the rock samples were powdered in agate ball mills at the Geological Survey of Japan. One of the splits from each unconsolidated sample was powdered in an agate puck mill at Memorial University of Newfoundland (MUN), with additional grinding of some samples by means of an artificial sapphire mortar and pestle.

All chemical analyses were performed at MUN and are reported either as weight percents (\%) or parts per million (ppm). Major elements were determined for the 271 samples using a Perkin Elmer model 2380 Atomic Absorption (AA) Spectrometer and calibration curves determined with suitable rock standards. Trace elements were determined for 253 pressed powder pellets $(5 \mathrm{~g}$ rock powder and $0.7 \mathrm{~g}$ phenolic acid, prebaked at $200^{\circ} \mathrm{C}$ ) by X-ray fluorescence (XRF), using an ARL model $8420+$ spectrometer. A subset of the trace elements, including the REEs, were determined on 16 samples by inductively coupled, plasma-source mass spectrometry (ICP-MS), using methods outlined by Jenner et al. (1990).

Precisions and detection limits for the analytical techniques at MUN are presented in Table 1. For AA, precision is based on eight replicates of a single volcanic rock of acid andesite composition. For XRF, concentrations are calibrated to rock standards BHVO-1 (Sc, $\mathrm{Ti}, \mathrm{V}, \mathrm{Cr}, \mathrm{Ni}, \mathrm{Cu}, \mathrm{Zn}$ ) and $\mathrm{SY}-2$ (Ga, Rb, Sr, Y, Zr, Ba, Ce, Pb). For ICP-MS, detection limits are calculated as three times the standard deviation of six replicate analyses of the calibration blank $(0.2 \mathrm{M}$ nitric acid). ICP-MS precisions, based on 54 analyses of USGS rock standards BIR-1, DNC-1, W-2, AGV-1, BCR-1, BHVO-1, and W-1 (Jenner et al., 1990), are presented as percentages of the concentration

Table 1. Analytical precision and normalization values for N-MORB and primitive mantle.

\begin{tabular}{|c|c|c|c|c|c|c|c|c|}
\hline \multirow[b]{2}{*}{ Species } & \multirow[b]{2}{*}{ Method } & \multirow[b]{2}{*}{ Unit } & \multirow[b]{2}{*}{$\begin{array}{l}\text { Standard } \\
\text { deviation }\end{array}$} & \multicolumn{5}{|c|}{ Values used to normalize plots } \\
\hline & & & & $\begin{array}{c}\text { Detection } \\
\text { limit }\end{array}$ & N-MORB & Ref. & $\begin{array}{l}\text { Primitive } \\
\text { mantle }\end{array}$ & Ref. \\
\hline $\mathrm{SiO}_{2}$ & AA & $\%$ & 0.297 & 0.891 & - & - & & \\
\hline $\mathrm{TiO}_{2}$ & AA & $\%$ & 0.067 & 0.201 & $=$ & $=$ & & \\
\hline $\mathrm{Al}_{2} \mathrm{O}_{3}$ & $\mathrm{AA}$ & $\%$ & 0.146 & 0.438 & - & - & & \\
\hline $\mathrm{MnO}$ & $\mathrm{AA}$ & $\%$ & $<0.005$ & $<0.015$ & - & - & & \\
\hline $\mathrm{MgO}$ & $\mathrm{AA}$ & $\%$ & 0.030 & 0.090 & - & - & & \\
\hline $\mathrm{CaO}$ & $\mathrm{AA}$ & $\%$ & 0.048 & 0.144 & $=$ & $=$ & & \\
\hline $\mathrm{Na}_{2} \mathrm{O}$ & $\mathrm{AA}$ & $\%$ & 0.016 & 0.048 & 2.679 & 4 & - & \\
\hline $\mathrm{K}_{2} \mathrm{O}$ & $\mathrm{AA}$ & $\%$ & 0.063 & 0.189 & 0.072 & i & - & \\
\hline $\mathrm{P}_{2} \mathrm{O}_{5}$ & $\mathrm{AA}$ & $\%$ & ND & ND & 0.117 & $\mathrm{i}$ & - & \\
\hline $\mathrm{Fe}_{2} \mathrm{O}_{3}$ & AA & $\%$ & 0.118 & 0.354 & - & - & & \\
\hline $\mathrm{Se}$ & XRF & ppm & 2 & 6 & - & - & & \\
\hline $\mathrm{Ti}$ & $\hat{X R F}$ & $\mathrm{ppm}$ & 2 & 7 & 7600.00 & I & 1085.10 & 4 \\
\hline $\mathrm{v}$ & $\hat{X R F}$ & $\mathrm{ppm}$ & 2 & 6 & 380.00 & 2 & - & \\
\hline $\mathrm{Cr}_{\mathrm{r}}$ & $\hat{X R F}$ & $\mathrm{ppm}$ & 2 & 5 & 307.00 & 3 & $\overline{-}$ & \\
\hline $\mathrm{Ni}$ & $\hat{X R F}$ & ppm & 2 & 5 & 149.50 & 4 & $\overline{-}$ & \\
\hline $\mathrm{Cu}$ & $\hat{X R F}$ & $\mathrm{ppm}$ & i & 4 & - & - & & \\
\hline $\mathrm{Zn}$ & $\mathrm{XRF}$ & $\mathrm{ppm}$ & i & 3 & - & - & & \\
\hline $\mathrm{Ga}$ & $\mathrm{XRF}$ & $\mathrm{ppm}$ & i & 3 & - & - & & \\
\hline $\mathrm{Rb}$ & $\hat{X R F}$ & $\mathrm{ppm}$ & i & 3 & 0.56 & 1 & - & \\
\hline $\mathrm{Sr}_{\mathrm{v}}$ & $\mathrm{XRF}$ & ppm & i & 3 & 90.00 & i & $\overline{-}$ & \\
\hline $\mathrm{Y}$ & $\mathrm{XRF}$ & $\mathrm{ppm}$ & $\mathrm{i}$ & 3 & 28.00 & i & 3.9400 & 4 \\
\hline $\mathrm{Zr}$ & $\hat{X R F}$ & ppm & $i$ & 3 & 74.00 & i & 9.7140 & 4 \\
\hline $\mathrm{Ba}$ & $\hat{X} \hat{R F}^{\prime}$ & ppm & 7 & $2 i$ & 6.30 & i & - & \\
\hline $\mathrm{Ce}$ & $\hat{X R F}$ & ppm & 9 & 26 & 7.50 & i & 1.6011 & 4 \\
\hline $\mathrm{Pb}$ & $\hat{X R F}$ & ppm & 2 & 20 & - & 1 & 1.0001 & \\
\hline Sc & ICP-MS & $\mathrm{ppm}$ & $3 \%-7 \%$ & 3.387 & - & - & & \\
\hline $\mathrm{Rb}$ & ICP-MS & ppm & $3 \%-7 \%$ & 1.110 & 0.56 & 1 & - & \\
\hline $\mathrm{Sr}_{\mathrm{S}}$ & ICP-MS & $\mathrm{ppm}$ & $<3 \%$ & 0.134 & 90.00 & i & $\overline{-}$ & \\
\hline$\vec{Y}$ & ICP-MS & ppm & $<3 \%$ & 0.010 & 28.00 & i & $\overline{3} 9400$ & 4 \\
\hline $\mathrm{Zr}$ & ICP-MS & ppont & $<3 \%$ & 0.242 & 74.00 & i & 9.7140 & 4 \\
\hline $\mathrm{Nb}$ & ICP-MS & $\mathrm{ppm}$ & $3 \%-7 \%$ & 0.011 & 2.33 & i & 0.6175 & 4 \\
\hline $\mathrm{Ba}$ & ICP-MS & $\mathrm{ppm}$ & $<3 \%$ & 0.389 & 6.30 & i & - & \\
\hline $\mathrm{Pb}$ & ICP-MS & $\begin{array}{l}\mathrm{ppm} \\
\mathrm{ppm}\end{array}$ & $3 \%-7 \%$ & 0.203 & -0.00 & 1 & - & \\
\hline Th & ICP-MS & $\mathrm{ppm}$ & $<3 \%$ & 0.064 & 0.12 & 1 & 0.0813 & 4 \\
\hline u & ICP-MS & $\mathrm{ppm}$ & $3 \%-7 \%$ & 0.036 & - & - & & \\
\hline $\mathrm{Li}$ & ICP-MS & $\mathrm{ppm}$ & $3 \%-7 \%$ & 0.948 & $=$ & $=$ & & \\
\hline $\mathrm{La}$ & ICP-MS & $\mathrm{ppm}$ & $<3 \%$ & 0.045 & 2.50 & 1 & 0.6139 & 4 \\
\hline $\mathrm{Ce}$ & ICP-MS & $\mathrm{ppm}$ & $<3 \%$ & 0.032 & 7.50 & i & 1.6011 & $\overrightarrow{4}$ \\
\hline Pr & ICP-MS & $\mathrm{ppm}$ & $<3 \%$ & 0.022 & 1.32 & i & 0.2419 & 4 \\
\hline $\mathrm{Nd}$ & ICP-MS & $\mathrm{ppm}$ & $<3 \%$ & 0.073 & 7.30 & i & 1.1892 & 4 \\
\hline $\mathrm{Sm}$ & ICP-MS & ppm & $<3 \%$ & 0.066 & 2.63 & i & 0.3865 & 4 \\
\hline Eu & ICP-MS & ppm & $3 \%-7 \%$ & 0.039 & 1.02 & i & 0.1456 & 4 \\
\hline Gd & ICP-MS & ppm & $<3 \%$ & 0.073 & 3.68 & i & 0.5128 & 4 \\
\hline $\mathrm{Tb}$ & ICP-MS & $\mathrm{ppm}$ & $<3 \%$ & 0.007 & - & - & & \\
\hline Dy & ICP-MS & $\mathrm{ppm}$ & $<3 \%$ & 0.025 & 4.55 & 1 & 0.6378 & 4 \\
\hline Ho & ICP-MS & $\mathrm{ppm}$ & $<3 \%$ & 0.003 & - & - & & \\
\hline Er & ICP-MS & $\mathrm{ppm}$ & $<3 \%$ & 0.048 & 2.97 & 1 & 0.4167 & 4 \\
\hline $\mathrm{Tm}$ & ICP-MS & $\mathrm{ppm}$ & $<3 \%$ & 0.008 & - & - & & \\
\hline $\mathrm{Yb}$ & ICP-MS & ppm & $<3 \%$ & 0.041 & 3.05 & 1 & 0.4144 & 4 \\
\hline Lu & ICP-MS & $\mathrm{ppm}$ & $3 \%-7 \%$ & 0.008 & 0.46 & i & 0.0637 & 4 \\
\hline $\begin{array}{l}\mathrm{Lu} \\
\mathrm{Hf}\end{array}$ & ICP-MS & $\begin{array}{l}\mathrm{ppm} \\
\mathrm{ppm}\end{array}$ & $<3 \%$ & $\begin{array}{l}0.068 \\
0.062\end{array}$ & 0.46 & 1 & & \\
\hline $\mathrm{Ta}_{\mathrm{T}}$ & ICP-MS & $\mathrm{ppm}$ & $-11 \%$ & 0.007 & - & & 0.0351 & 4 \\
\hline
\end{tabular}


in the sample. These values are valid down to concentrations of about ten times the detection limit. ICP-MS accuracies, as the percent difference from published concentrations in standards AGV-1, BCR-1, BHVO-1, and $\mathrm{W}-1$, range from $<3 \%(\mathrm{Li}, \mathrm{Cs}, \mathrm{Rb}, \mathrm{Sr}, \mathrm{Ba}, \mathrm{Hf}, \mathrm{Ta}, \mathrm{Sc}$, $\mathrm{La}, \mathrm{Ce}, \mathrm{Pr}, \mathrm{Nd}, \mathrm{Gd}, \mathrm{Tb}, \mathrm{Er}, \mathrm{Yb}, \mathrm{U}, \mathrm{Pb})$, to $3 \%-7 \%$ (Zr, Nb, Y, Sm, Eu, Dy, Lu), to $7 \%-12 \%$ (Ho, Th, Tm).

For those elements determined both by XRF and ICP-MS (Sc, V, $\mathrm{Rb}, \mathrm{Sr}, \mathrm{Y}, \mathrm{Zr}, \mathrm{Nb}, \mathrm{Ba}, \mathrm{Pb}, \mathrm{Th}, \mathrm{U}$, and $\mathrm{Ce} ; 16$ samples only), excellent agreement exists between methods for most elements well above their detection limits ( $\mathrm{Sc}, \mathrm{Sr}, \mathrm{Y}, \mathrm{Zr}$ ), and poor or indeterminate agreement for elements in abundances near their XRF detection limits ( $\mathrm{Rb}, \mathrm{Nb}$, $\mathrm{Ba}, \mathrm{Pb}, \mathrm{Th}, \mathrm{U}, \mathrm{Ce})$. We do not report XRF determinations of the uniformly low concentrations of $\mathrm{Th}, \mathrm{U}$, and $\mathrm{Nb}$, which are close to or below detection limits. Low ICP-MS concentrations for Be, Mo, Tl, $\mathrm{Cs}$, and $\mathrm{Bi}$, and consistently lower estimates of $\mathrm{V}$ by ICP-MS than by $\mathrm{XRF}$, lead us to suspect the ICP-MS data for these elements; therefore, these values are not reported in this paper. Some elements determined by the MUN ICP-MS facility are not referred to in this paper, but the data are tabulated for use by other researchers.

Loss on ignition (LOI) is, in almost all cases, a result of release of bound water; values as great as about $20 \%$ in the Leg 126 sandstone samples are characteristic of smectite-rich, diagenetically altered sandstones (Egeberg and the Leg 126 Shipboard Scientific Party, 1990). In a few samples, high $\mathrm{LOI}$, high $\mathrm{CaO}$, and an indication from shipboard analyses of more than about $1 \% \mathrm{CaCO}_{3}$ (Taylor, Fujioka, et al., 1990) prompted us to measure $\mathrm{CaCO}_{3}$ content using the method outlined below. Because micropaleontological data and shipboard descriptions suggest that all calcite in these rocks is biogenic, the calcite $\mathrm{CaO}$ was subtracted from bulk $\mathrm{CaO}$ abundances for determination of the primary element ratios in the volcanic source rocks.

Calcite was determined by digestion of $0.1000 \mathrm{~g}$ of rock powder in $10 \mathrm{ml}$ of $25 \%$ glacial acetic for $24 \mathrm{hr}$ following $30 \mathrm{~min}$ of mechanical shaking. The filtrate was then diluted to $200 \mathrm{ml}$ and analyzed for $\mathrm{CaO}$ by $\mathrm{AA}$; the measurement was then converted to an equivalent percentage of $\mathrm{CaCO}_{3}$ in the bulk sample. For samples from intervals where shipboard $\mathrm{CaCO}_{3}$ analyses exist, results are in close agreement with the shipboard data (the difference is less than one percentage point for $\mathrm{CaCO}_{3}<5 \%$, and about three percentage points for $\mathrm{CaCO}_{3}=40 \%$ in one anomalously calcareous sample).

\section{MAJOR ELEMENTS}

All analytical results are reported in Table 2 as volatile-free percentages; that is, the data are recalculated to $100 \%$ ignoring LOI and calcite $\mathrm{CaO}$ (i.e., biogenic calcite). The analyses are ordered first by site and then by age, although in the variation diagrams that follow, samples are sometimes discriminated by age but never by site. $\mathrm{CaCO}_{3}$ content, where determined, is reported in the table.

Potassium and sodium (both rock and seawater sodium) are known to be particularly mobile, both during weathering and later burial of volcanic materials (Nesbitt and Young, 1989). In plots of alkali metals against silica, significant scatter is present in samples older than $20 \mathrm{Ma}$. For younger material, however, primary alkali contents appear to be reflected more accurately and the data points cluster along igneous trends. In particular, for samples younger than $20 \mathrm{Ma}$, the source rocks appear to have been uniformly subalkaline, mainly low-potassium volcanic rocks (Fig. 3). As a test of whether weathering may have rendered the alkali content of the detritus significantly different from that in the source volcanic rocks, we calculated the molar ratio $\mathrm{Al}_{2} \mathrm{O}_{3} /\left(\mathrm{Al}_{2} \mathrm{O}_{3}+\mathrm{CaO}+\mathrm{Na}_{2} \mathrm{O}+\mathrm{K}_{2} \mathrm{O}\right)$ for all samples younger than $20 \mathrm{Ma}$. According to Nesbitt and Young (1989), this molar ratio is about 0.5 for unweathered basalts and granodiorites, and higher for weathered materials. The mean and standard deviation of the molar ratio for the Izu-Bonin Arc samples is $0.45 \pm 0.03$, consistent with negligible preburial weathering of the detritus.

The $\mathrm{FeO} / \mathrm{MgO}$ ratios (Fig. 4) imply that the igneous source rocks were both tholeiitic and calc-alkaline, but this may be an artifact of

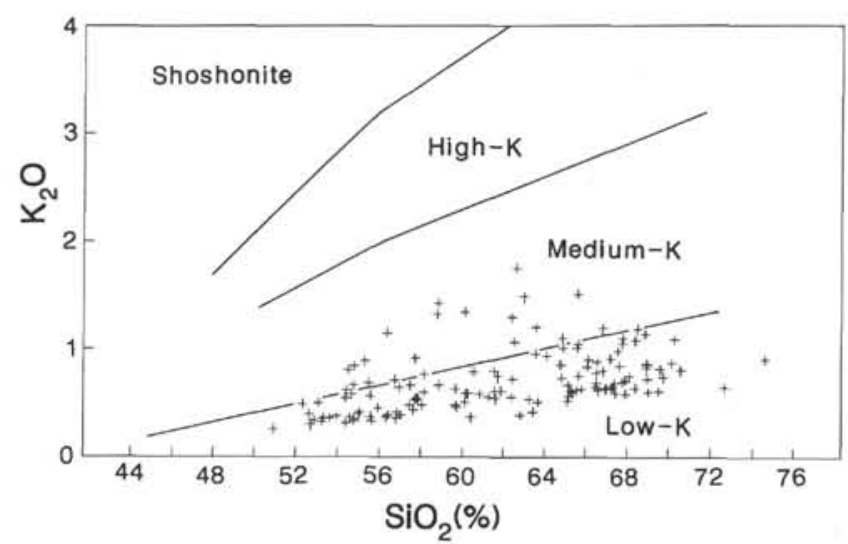

Figure 3. Potash vs. silica for samples younger than $20 \mathrm{Ma}$. Older samples scatter up to values of about $4 \%$ potash. This scatter is ascribed to diagenesis. The dividing lines between fields are from Peccerillo and Taylor (1976).

mechanical mixing of variously differentiated tholeiitic rocks during sediment transport. Almost all samples have silica contents of $50 \%-$ $70 \%$ (basaltic to rhyolitic material), with a preponderance of andesitic compositions (Fig. 5). Very few samples exceed $70 \% \mathrm{SiO}_{2}$, in contrast to analyses of individual pumice clasts and glass shards (Gill et al., this volume; Rodolfo et al., this volume), some of which have volatile-free silica contents of more than $75 \%$. MgO contents, which reach values of about $10 \%$ in the nearby Eocene boninites (Hickey and Frey, 1982), only exceed $7.0 \%$ in 17 samples. These have basalt and basic andesite compositions (Fig. 6), and are mostly older than $28.5 \mathrm{Ma}$ and from the forearc basin (Fig. 7). Only one sample with

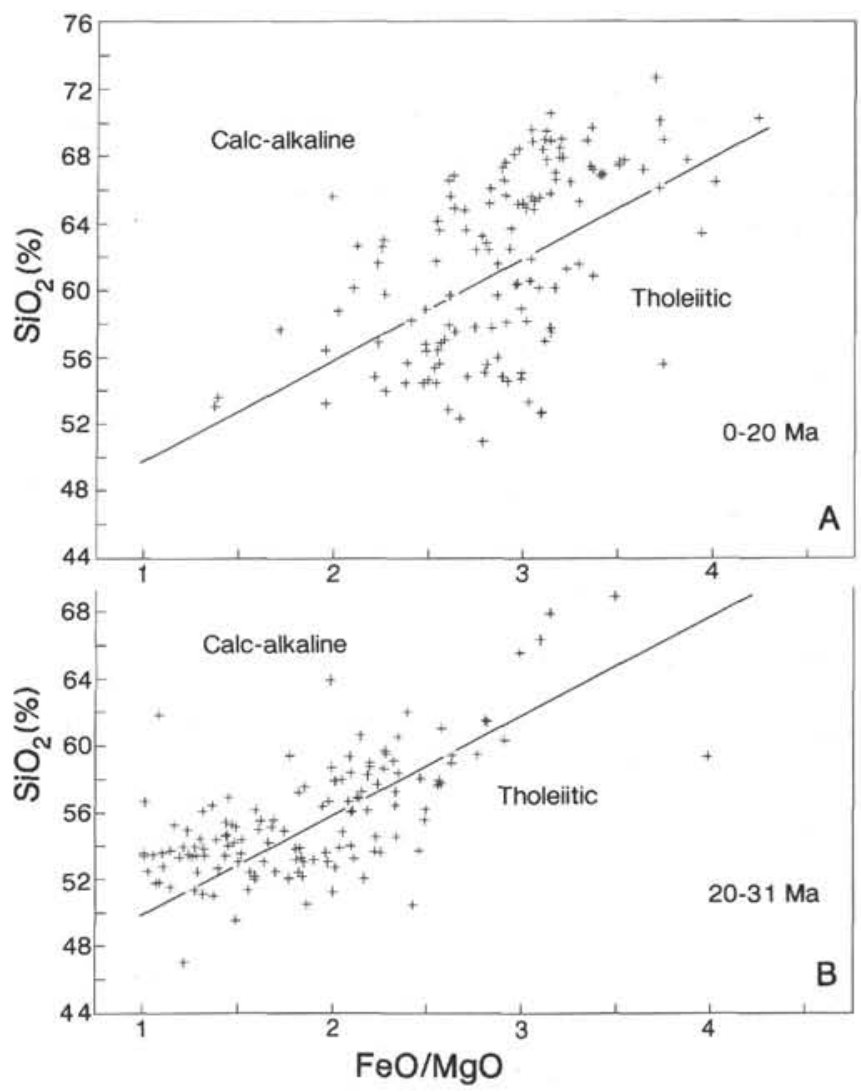

Figure 4. Silica vs. FeO/MgO. A. 0-20 Ma. B. 20-31 Ma. The dividing line between tholeiitic and calc-alkaline fields is from Miyashiro (1974). 


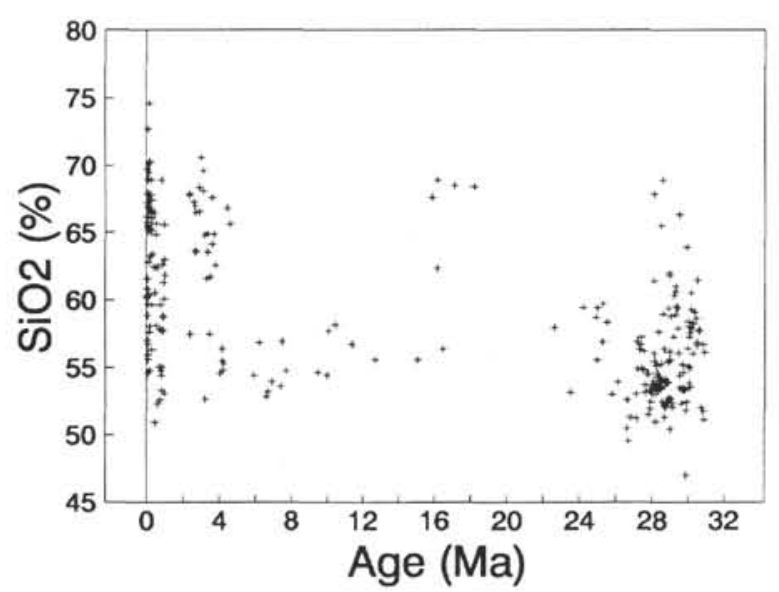

Figure 5. Silica vs. age, for all samples. Age is taken from age-depth plots in Taylor, Fujioka, et al. (1990).

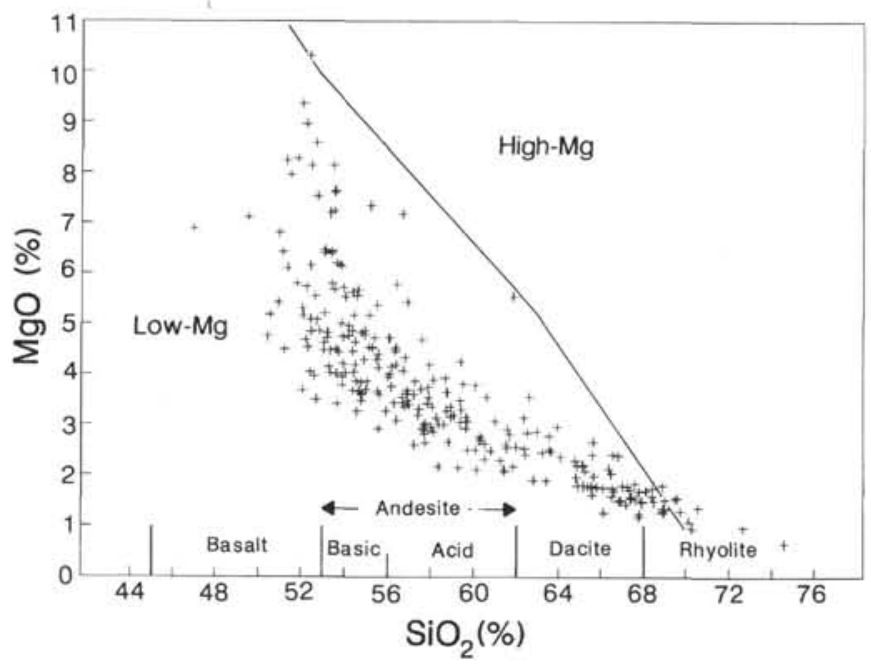

Figure 6. $\mathrm{MgO}$ vs. silica, for all samples. The $\mathrm{SiO}_{2}$-based boundaries between volcanic rock types are those of Taylor (1969). The boundary between low- and high-Mg fields is justified in Taylor, Fujioka, et al. (1990).

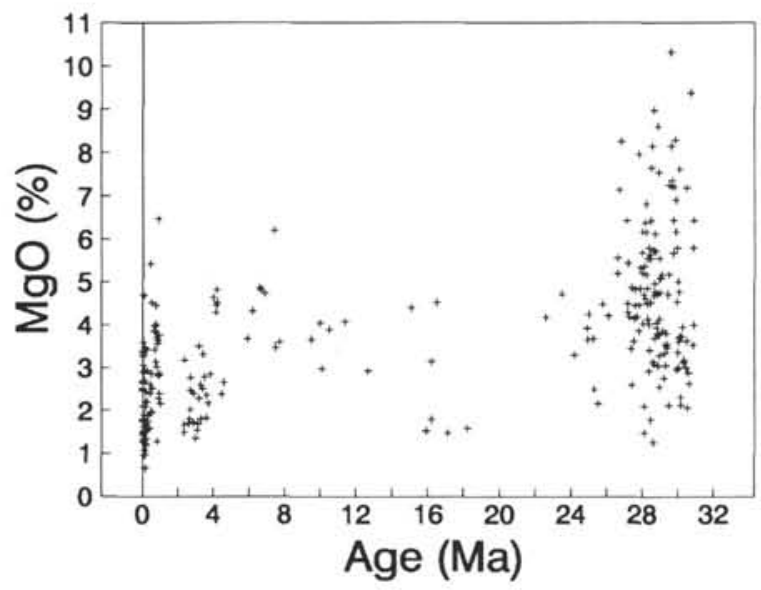

Figure 7. $\mathrm{MgO}$ vs. age, for all samples. Age is taken from age-depth plots in Taylor, Fujioka, et al. (1990). less than $68 \% \mathrm{SiO}_{2}$ falls within the field of high-MgO lavas (Fig. 6), as defined by the shipboard petrologists (Taylor, Fujioka, et al., 1990).

The major element geochemistry gives a general impression that the source rocks consisted of calc-alkaline and tholeiitic basalts to rhyolites of island-arc character. Volcanic source rocks were more basic than average (lower $\mathrm{SiO}_{2}$, higher $\mathrm{MgO}$ ) during the Oligocene, and more acid than average since about $4 \mathrm{Ma}$. The major element chemistry of single mineral phases, like orthopyroxene and clinopyroxene, also indicates calc-alkaline to tholeiitic, island-arc source compositions (Fujioka and Saito, this volume).

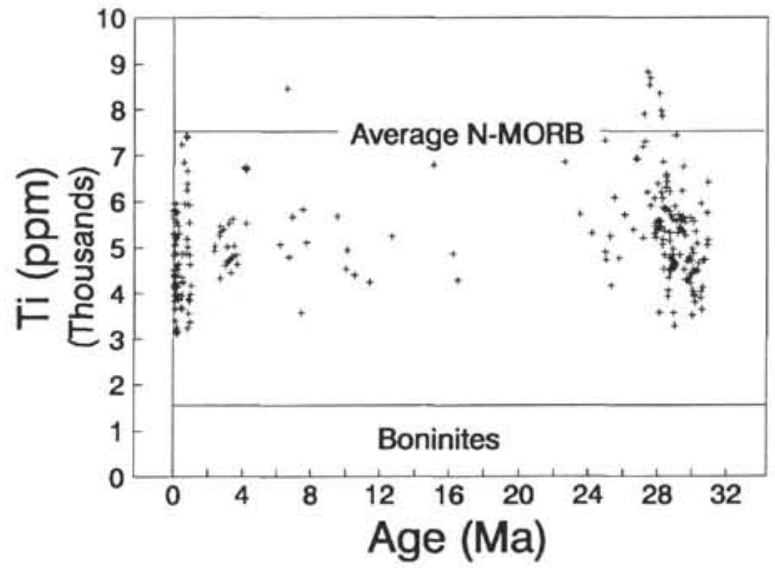

Figure 8. Titanium content vs. age, for all samples. Age is taken from age-depth plots in Taylor, Fujioka, et al. (1990). Ti concentration in average N-MORB and boninites, respectively, is $7600 \mathrm{ppm}$ (Table 1) and less than about 1500 ppm (Murton, 1989).

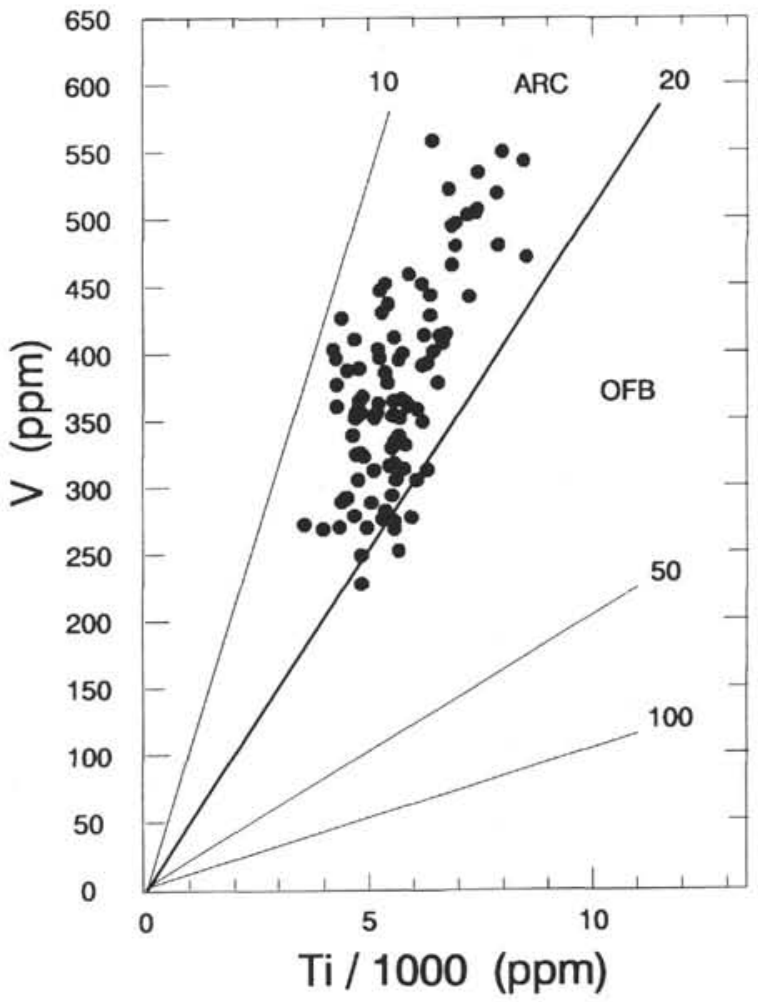

Figure 9. Vanadium vs. titanium, with the dividing line between fields for volcanic arcs (ARC) and ocean-floor basalts (OFB) from Shervais (1982). Only 109 samples with the composition of basalts and basic andesites $(45 \%-$ $56 \% \mathrm{SiO}_{2}$ ) are plotted. 
Table 2. Volatile-free major element data.

\begin{tabular}{|c|c|c|c|c|}
\hline & & & & \\
\hline interval $(\mathrm{cm})$ & (mbsf) & (Ma) & $\mathrm{SiO}_{2}$ & $\mathrm{TiO}_{2}$ \\
\hline 7 & & & & \\
\hline $787 \mathrm{~A}-1 \mathrm{~W}-1,26-32$ & 0.26 & 0.01 & 60.14 & 0.64 \\
\hline 787B-1R-1, $48-52$ & 0.48 & 0.01 & 57.01 & 0.81 \\
\hline $787 \mathrm{~B}-2 \mathrm{R}-1,6-10$ & 2.36 & 0.04 & 54.60 & 0.71 \\
\hline 787A-1W-3, 15-21 & 3.15 & 0.05 & 56.80 & 0.73 \\
\hline 787B-2R-1, $103-107$ & 3.33 & 0.06 & 55.62 & 0.77 \\
\hline $787 \mathrm{~B}-3 \mathrm{R}-2,76-79$ & 14.16 & 0.25 & 56.31 & 0.77 \\
\hline 787B-7R-1, 3-7 & 49.83 & 24.20 & 59.46 & 0.73 \\
\hline 787B-9R-2, $47-50$ & 70.97 & 25.00 & 59.44 & 0.59 \\
\hline $787 \mathrm{~B}-14 \mathrm{R}-2,69-73$ & 119.69 & 26.70 & 49.53 & 0.83 \\
\hline $787 \mathrm{~B}-14 \mathrm{R}-3,72-76$ & 120.93 & 26.80 & 51.32 & 0.83 \\
\hline 787B-15R-1, 108-111 & 128.28 & 27.10 & 53.05 & 0.84 \\
\hline $787 \mathrm{~B}-15 \mathrm{R}-3,52-55$ & 130.67 & 27.15 & 56.91 & 0.76 \\
\hline $787 \mathrm{~B}-15 \mathrm{R}-4,26-29$ & 131.91 & 27.18 & 54.93 & 0.92 \\
\hline $787 \mathrm{~B}-16 \mathrm{R}-1,46-49$ & 137.26 & 27.25 & 56.38 & 0.79 \\
\hline $787 \mathrm{~B}-17 \mathrm{R}-4,10-13$ & 151.10 & 27.35 & 56.69 & 0.96 \\
\hline $787 \mathrm{~B}-17 \mathrm{R}-5,92-95$ & 152.71 & 27.40 & 57.25 & 1.14 \\
\hline $787 \mathrm{~B}-18 \mathrm{R}-1,17-20$ & 156.37 & 27.50 & 54.84 & 1.09 \\
\hline 787B-18R-3, $142-145$ & 160.02 & 27.55 & 56.20 & 0.96 \\
\hline $787 \mathrm{~B}-19 \mathrm{R}-3,31-34$ & 169.11 & 27.60 & 53.72 & 1.13 \\
\hline 787B-19R-6, $45-48$ & 173.75 & 27.70 & 54.53 & 0.84 \\
\hline 787B-20R-1, 15-16 & 175.65 & 27.80 & 54.55 & 1.01 \\
\hline 787B-20R-4, 20-23 & 180.20 & 27.85 & 53.32 & 0.73 \\
\hline $787 \mathrm{~B}-21 \mathrm{R}-1,65-69$ & 185.75 & 27.87 & 52.02 & 0.63 \\
\hline $787 \mathrm{~B}-22 \mathrm{R}-1,47-50$ & 195.27 & 27.90 & 52.46 & 0.64 \\
\hline $787 \mathrm{~B}-23 \mathrm{R}-4,18-21$ & 209.08 & 28.00 & 53.04 & $\begin{array}{l}0.64 \\
0.69\end{array}$ \\
\hline $787 \mathrm{~B}-24 \mathrm{R}-6,91-94$ & 222.51 & 28.08 & 61.43 & 0.74 \\
\hline $1,119-122$ & 224.89 & 28.10 & 67.92 & 0.44 \\
\hline $787 \mathrm{~B}-25 \mathrm{~K}$ & 228.30 & 28.12 & 55.52 & 0.69 \\
\hline 787B-26R-5, 19-22 & 239.59 & 28.28 & 53.62 & 0.71 \\
\hline 787B-27R-1,77-80 & 243.77 & 28.30 & 53.26 & 0.71 \\
\hline $787 \mathrm{~B}-27 \mathrm{R}-\mathrm{CC}, 0-3$ & 248.12 & 28.38 & 53.62 & 0.76 \\
\hline $787 \mathrm{~B}-29 \mathrm{R}-2,77-80$ & 264.67 & 28.45 & 65.53 & 0.64 \\
\hline $787 \mathrm{~B}-30 \mathrm{R}-3,62-65$ & 275.62 & 28.58 & 68.92 & 0.56 \\
\hline 787B-31R-1, $135-138$ & 283.05 & 28.63 & 52.20 & 0.67 \\
\hline $787 \mathrm{~B}-32 \mathrm{R}-1,54-57$ & 291.84 & 28.70 & 51.34 & 0.66 \\
\hline $787 \mathrm{~B}-33 \mathrm{R}-2,68-71$ & 302.16 & 28.80 & 52.40 & 0.71 \\
\hline $788 \mathrm{C}-2 \mathrm{H}-1,6-10$ & 13.36 & 0.10 & 66.94 & 0.79 \\
\hline $788 \mathrm{C}-3 \mathrm{H}-1,42-46$ & 20.62 & 0.15 & 66.60 & 0.75 \\
\hline $788 \mathrm{~A}-4 \mathrm{R}-1,18-21$ & 26.38 & 0.20 & 67.43 & 0.92 \\
\hline $788 \mathrm{C}-4 \mathrm{H}-1,64-67$ & 30.34 & 2.35 & 67.92 & 0.75 \\
\hline $788 \mathrm{C}-4 \mathrm{H}-4,46-49$ & 34.66 & 2.36 & 67.77 & 0.67 \\
\hline $788 \mathrm{C}-7 \mathrm{H}-1,30-33$ & 58.50 & 2.58 & 67.31 & 0.68 \\
\hline $788 \mathrm{C}-8 \mathrm{H}-1,20-23$ & 67.90 & 2.64 & 67.01 & 0.75 \\
\hline $788 \mathrm{C}-8 \mathrm{H}-2,107-110$ & 70.27 & 2.68 & 66.47 & 0.75 \\
\hline $788 \mathrm{C}-9 \mathrm{H}-1,85-88$ & 78.05 & 2.71 & 63.67 & 0.79 \\
\hline $788 \mathrm{C}-11 \mathrm{H}-3,125-128$ & 100.45 & 2.88 & 66.56 & 0.76 \\
\hline $788 \mathrm{C}-11 \mathrm{H}-\mathrm{CC}, 13-16$ & 102.53 & 2.89 & 68.38 & 0.76 \\
\hline $788 \mathrm{C}-13 \mathrm{H}-5,120-124$ & 122.40 & 2.98 & 70.57 & 0.63 \\
\hline $788 \mathrm{C}-14 \mathrm{H}-5,57-60$ & 131.27 & 3.10 & 69.59 & 0.63 \\
\hline $788 \mathrm{C}-15 \mathrm{H}-1,5-9$ & 134.25 & 3.12 & 68.09 & 0.67 \\
\hline $788 \mathrm{C}-16 \mathrm{H}-1,7-11$ & 143.77 & 3.19 & 64.78 & 0.80 \\
\hline $788 \mathrm{C}-17 \mathrm{H}-4,94-98$ & 158.64 & 3.28 & 61.54 & 0.84 \\
\hline $788 \mathrm{C}-18 \mathrm{H}-2,65-68$ & 164.85 & 3.31 & 64.92 & 0.63 \\
\hline $788 \mathrm{C}-19 \mathrm{H}-1,68-71$ & 172.88 & 3.38 & 63.55 & 0.71 \\
\hline $788 \mathrm{C}-20 \mathrm{H}-2,40-43$ & 183.60 & 3.44 & 57.47 & 0.99 \\
\hline$-21 \mathrm{H}-1,40-43$ & $\begin{array}{l}193.60 \\
191.6\end{array}$ & 3.50 & 61.70 & 0.83 \\
\hline $8 \mathrm{C}-22 \mathrm{H}-1,6-9$ & 200.76 & 3.58 & $\begin{array}{l}67.57 \\
67.70\end{array}$ & 0.72 \\
\hline $88 \mathrm{C}-23 \mathrm{H}$ & 210.66 & 3.63 & 64.13 & 0.76 \\
\hline $110-114$ & 222.30 & 3.70 & 64.88 & $\begin{array}{l}0.10 \\
0.76\end{array}$ \\
\hline $788 \mathrm{C}-26 \mathrm{I}$ & 238.76 & 3.82 & $\begin{array}{l}62.53 \\
62.83\end{array}$ & 0.67 \\
\hline $788 \mathrm{D}-5 \mathrm{R}-1,42-45$ & 258.62 & $\begin{array}{l}3.82 \\
4.02\end{array}$ & $\begin{array}{l}02 . \\
54 .\end{array}$ & $\begin{array}{l}0.07 \\
1.00\end{array}$ \\
\hline $788 \mathrm{D}-6 \mathrm{R}-1,24-27$ & 268.14 & 4.14 & 55. & 0.96 \\
\hline c. $7-10$ & 279 & 4.17 & 56 & 0.8 \\
\hline $29-32$ & 287.49 & 4.20 & 54 & 0. \\
\hline $\mathrm{R}-1,101-105$ & 297.91 & 4.23 & 55. & 0.89 \\
\hline $11 \mathrm{R}-1,17-20$ & 316.17 & 4.44 & 66 & 0.72 \\
\hline $1,17-20$ & 325.77 & 4.57 & 65 & 0. \\
\hline$-1 \mathrm{H}-5,132-135$ & 7.32 & 0.01 & 66 & 0. \\
\hline $9-1$ & 14.89 & 0.02 & 61.55 & 0.94 \\
\hline$-3 \mathrm{H}-2,21-24$ & 15.71 & 0.02 & 60.27 & 1.00 \\
\hline$-2 \mathrm{H}-6,106-109$ & 17.36 & 0.02 & 58.85 & 0.85 \\
\hline$-4 \mathrm{H}-3,47-50$ & 31.27 & 0.03 & 65 . & 0.76 \\
\hline $00 \mathrm{~B}-4 \mathrm{H}-6,74-77$ & 31.74 & 0.03 & 67.92 & 0.64 \\
\hline $0 \mathrm{~B}-5 \mathrm{H}-1,113-116$ & 34.13 & 0.04 & 59.72 & 0.69 \\
\hline $6 \mathrm{H}-4,16-20$ & 47.16 & 0.05 & 65.36 & 0.66 \\
\hline $0 \mathrm{~B}-7 \mathrm{H}-6,86-90$ & 60.36 & 0.07 & 68. & \\
\hline $0 \mathrm{~B}-8 \mathrm{H}-5,118-121$ & $\begin{array}{l}68.78 \\
68.78\end{array}$ & 0.07 & 55. & 0.89 \\
\hline $97-100$ & 73.77 & 0.08 & 69. & 0.68 \\
\hline $790 \mathrm{~B}-10 \mathrm{H}-6,119-122$ & 89.69 & 0.09 & 62. & 0.71 \\
\hline $790 \mathrm{C}-1 \mathrm{H}-4,30-33$ & 89.80 & 0.10 & 69. & 0.60 \\
\hline $790 \mathrm{~B}-11 \mathrm{H}-1,104-107$ & 91. & 0.10 & 70. & 0.54 \\
\hline $790 \mathrm{C}-5 \mathrm{H}$ & 128. & 0.15 & 67 & 0.54 \\
\hline & & 0. & & 0.44 \\
\hline 79 & & & & 0. \\
\hline 77 & & 0. & & 0 \\
\hline $44-47$ & $t$ & & 65 & 0. \\
\hline $81-8$ & 185. & 0.28 & & 0 . \\
\hline $790 \mathrm{C}-15 \mathrm{X}-\mathrm{CC}, 12-14$ & 213.49 & 0.43 & 60.54 & 0.62 \\
\hline 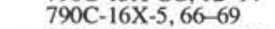 & 229.46 & 0.55 & 62.39 & 0.65 \\
\hline $790 \mathrm{C}-20 \mathrm{X}-\mathrm{CC}, 31-34$ & 266.52 & 0.94 & 65.60 & 0.58 \\
\hline $791 \mathrm{~A}-3 \mathrm{H}-1,83-86$ & 14.83 & 0.01 & 67.22 & 0.67 \\
\hline
\end{tabular}


Table 2 (continued).

\begin{tabular}{|c|c|c|c|}
\hline & & & \\
\hline interval $(\mathrm{cm})$ & (mbsf) & (Ma) & $\mathrm{SiO}_{2}$ \\
\hline 126-(Cont.) & & & \\
\hline $791 \mathrm{~A}-4 \mathrm{H}-2,142-145$ & 26.42 & 0.02 & 60.11 \\
\hline $791 \mathrm{~A}-5 \mathrm{H}-2,92-95$ & 35.42 & 0.02 & 60.83 \\
\hline $791 \mathrm{~A}-5 \mathrm{H}-6,53-56$ & 41.03 & 0.02 & 67.19 \\
\hline $791 \mathrm{~A}-8 \mathrm{H}-5,120-123$ & 68.80 & 0.03 & 69.71 \\
\hline $791 \mathrm{~A}-12 \mathrm{H}-3,106-110$ & 104.46 & 0.04 & 65.19 \\
\hline $791 \mathrm{~A}-15 \mathrm{H}-3,84-87$ & 133.34 & 0.06 & 65.55 \\
\hline $791 \mathrm{~A}-16 \mathrm{H}-5,73-76$ & 145.93 & 0.06 & $\begin{array}{l}0.353 \\
65.59\end{array}$ \\
\hline $791 \mathrm{~A}-18 \mathrm{H}-2,54-57$ & $\begin{array}{l}143.93 \\
160.54\end{array}$ & $\begin{array}{l}0.00 \\
0.07\end{array}$ & $\begin{array}{l}65.39 \\
66.84\end{array}$ \\
\hline $791 \mathrm{~A}-18 \mathrm{H}-7,15-18$ & $\begin{array}{l}100.34 \\
167.15\end{array}$ & 0.08 & $\begin{array}{l}60.84 \\
61.58\end{array}$ \\
\hline $791 \mathrm{~A}-20 \mathrm{H}-3,117-120$ & 181.87 & 0.08 & 68.97 \\
\hline $791 \mathrm{~A}-22 \mathrm{H}-5,128-131$ & 204.18 & 0.09 & 72.69 \\
\hline $791 \mathrm{~A}-28 \mathrm{X}-\mathrm{CC}, 8-11$ & 247.03 & 0.12 & $\begin{array}{l}12.69 \\
57.62\end{array}$ \\
\hline $791 \mathrm{~A}-34 \mathrm{X}-\mathrm{CC}, 12-15$ & 304.95 & 0.15 & 70.28 \\
\hline $791 \mathrm{~A}-41 \mathrm{X}-\mathrm{CC}, 1-7$ & 371.71 & 0.19 & 67.79 \\
\hline $791 \mathrm{~A}-46 \mathrm{X}-\mathrm{CC}, 8-13$ & 418.88 & 0.22 & 68.92 \\
\hline $791 \mathrm{~B}-11 \mathrm{R}-\mathrm{CC}, 13-16$ & 483.03 & 0.25 & 67.40 \\
\hline $791 \mathrm{~B}-15 \mathrm{R}-1,3-6$ & 521.53 & 0.29 & 66.06 \\
\hline $791 \mathrm{~B}-15 \mathrm{R}-\mathrm{CC}, 3-6$ & 521.63 & 0.29 & 66.54 \\
\hline $791 \mathrm{~B}-44 \mathrm{R}-1,21-24$ & 800.91 & 0.86 & 62.63 \\
\hline $791 \mathrm{~B}-44 \mathrm{R}-1,101-104$ & 801.71 & 0.87 & 58.75 \\
\hline $791 \mathrm{~B}-45 \mathrm{R}-2,56-59$ & 812.36 & 0.92 & 53.08 \\
\hline 791 B-45R-CC, $12-15$ & 813.57 & 0.92 & 58.82 \\
\hline $791 \mathrm{~B}-46 \mathrm{R}-1,72-75$ & 820.72 & 0.94 & 60.10 \\
\hline $791 \mathrm{~B}-47 \mathrm{R}-1,36-39$ & 829.96 & 0.96 & 62.98 \\
\hline $792 \mathrm{~A}-1 \mathrm{H}-6,52-55$ & $\begin{array}{r}89.020 \\
\end{array}$ & $\begin{array}{l}0.12 \\
0.12\end{array}$ & $\begin{array}{l}62.38 \\
60.39\end{array}$ \\
\hline $792 \mathrm{~A}-1 \mathrm{H}-6,95-99$ & 8.45 & 0.13 & 58.04 \\
\hline $792 \mathrm{~A}-2 \mathrm{H}-1,87-90$ & 10.57 & 0.14 & $\begin{array}{l}38.04 \\
54.79\end{array}$ \\
\hline $792 \mathrm{~A}-2 \mathrm{H}-4,46-50$ & 14.66 & 0.19 & $\begin{array}{l}34.79 \\
65.13\end{array}$ \\
\hline $792 \mathrm{~A}-3 \mathrm{H}-5,17-20$ & 25.27 & 0.32 & 63.41 \\
\hline $792 \mathrm{~A}-4 \mathrm{H}-5,14-17$ & 34.54 & 0.44 & 50.91 \\
\hline , $52-55$ & 39.82 & 0.50 & 65.66 \\
\hline $4.82-85$ & 52.52 & 0.65 & 55.05 \\
\hline $792 \mathrm{~B}-2 \mathrm{H}-3,145-148$ & 54.45 & 0.68 & 57.84 \\
\hline $792 \mathrm{~B}-2 \mathrm{H}-6,132-135$ & 58.41 & 0.75 & 54.77 \\
\hline $792 \mathrm{~B}-3 \mathrm{H}-3,26-30$ & 62.86 & 0.77 & 53.31 \\
\hline $792 \mathrm{~A}-7 \mathrm{H}-5,118-121$ & 63.45 & 0.78 & 54.40 \\
\hline $792 \mathrm{~B}-3 \mathrm{H}-4,90-93$ & 65.00 & 0.80 & 55.01 \\
\hline $792 \mathrm{~A}-8 \mathrm{H}-2,127-131$ & 68.64 & 0.82 & 57.76 \\
\hline $792 \mathrm{~A}-9 \mathrm{H}-2,60-63$ & 77.42 & 0.90 & 57.71 \\
\hline $792 \mathrm{~B}-5 \mathrm{X}-\mathrm{CC}, 25-28$ & 79.05 & 0.91 & 61.26 \\
\hline $792 \mathrm{~A}-10 \mathrm{H}-4,73-76$ & 89.34 & 1.00 & 61.85 \\
\hline $792 B-8 X-3,62-65$ & 111.52 & 2.39 & 57.44 \\
\hline $792 \mathrm{E}-1 \mathrm{R}-1,92-96$ & 136.52 & 2.70 & 63.58 \\
\hline $792 \mathrm{E}-4 \mathrm{R}-2,54-57$ & $\begin{array}{l}130.52 \\
166.54\end{array}$ & 3.20 & 52.69 \\
\hline $792 \mathrm{E}-7 \mathrm{R}-1,8-11$ & $\begin{array}{l}100.34 \\
193.48\end{array}$ & 5.90 & 54.40 \\
\hline $792 \mathrm{E}-8 \mathrm{R}-1,18-21$ & 203.28 & 6.20 & $\begin{array}{l}34.40 \\
56.83\end{array}$ \\
\hline $792 \mathrm{E}-9 \mathrm{R}-3,68-73$ & $\begin{array}{l}203.28 \\
216.28\end{array}$ & $\begin{array}{l}6.20 \\
6.60\end{array}$ & $\begin{array}{l}56.83 \\
52.84\end{array}$ \\
\hline $792 \mathrm{E}-9 \mathrm{R}-5,26-31$ & $\begin{array}{l}210.28 \\
217.86\end{array}$ & $\begin{array}{l}6.60 \\
6.70\end{array}$ & $\begin{array}{r}32.84 \\
53.22\end{array}$ \\
\hline $792 \mathrm{E}-11 \mathrm{R}-1,87-90$ & 232.77 & $\begin{array}{l}6.90 \\
6.90\end{array}$ & 53.95 \\
\hline $792 \mathrm{E}-12 \mathrm{R}-3,108-110$ & 245.58 & 7.40 & 53.61 \\
\hline 79 & 252.95 & 7.50 & 56.92 \\
\hline $19-23$ & 261.09 & 7.70 & 54.77 \\
\hline $792 \mathrm{E}-16 \mathrm{R}-2,93-97$ & 282.63 & 9.50 & 54.68 \\
\hline $792 \mathrm{E}-17 \mathrm{R}-2,46-50$ & 291.86 & 10.00 & 54.44 \\
\hline $792 \mathrm{E}-17 \mathrm{R}-3,22-25$ & 293.12 & 10.10 & 57.71 \\
\hline $792 \mathrm{E}-18 \mathrm{R}-2,43-46$ & 301.43 & 10.50 & 58.16 \\
\hline $792 \mathrm{E}-20 \mathrm{R}-3,55-58$ & 322.15 & 11.40 & 56.72 \\
\hline $792 \mathrm{E}-23 \mathrm{R}-1,118-121$ & 348.78 & 12.70 & 55.57 \\
\hline $792 \mathrm{E}-28 \mathrm{R}-2,94-97$ & 398.44 & 22.60 & 57.96 \\
\hline $792 \mathrm{E}-30 \mathrm{R}-1,116-119$ & 416 . & 24.90 & 58.72 \\
\hline $792 \mathrm{E}-31 \mathrm{R}-2,19-22$ & 426.59 & 25.50 & 58.38 \\
\hline $792 \mathrm{E}-32 \mathrm{R}-3,81-84$ & 438.41 & 26. & 53.94 \\
\hline $792 \mathrm{E}-33 \mathrm{R}-1,105-108$ & 445.25 & 27.40 & 54.95 \\
\hline $792 \mathrm{E}-34 \mathrm{R}-\mathrm{CC}, 20-23$ & 458.86 & 28.00 & 53.74 \\
\hline $792 \mathrm{E}-36 \mathrm{R}-1,30-34$ & $\begin{array}{l}438.00 \\
473.50\end{array}$ & 28. & 56.08 \\
\hline $792 \mathrm{E}-37 \mathrm{R}-4,57-6$ & 487.87 & 28.1 & $\begin{array}{l}30.08 \\
53.36\end{array}$ \\
\hline$-38 \mathrm{R}-5,50-$ & $\begin{array}{l}481.81 \\
499.00\end{array}$ & 28.20 & $\begin{array}{l}33.36 \\
53.86\end{array}$ \\
\hline $792 \mathrm{E}-38 \mathrm{R}-1,28-32$ & 502.48 & 28.22 & $\begin{array}{l}33.86 \\
55.14\end{array}$ \\
\hline $792 \mathrm{E}-40 \mathrm{R}-2,57-62$ & $\begin{array}{l}502.48 \\
513.97\end{array}$ & $\begin{array}{l}28.22 \\
28.28\end{array}$ & $\begin{array}{l}53.14 \\
54.60\end{array}$ \\
\hline $792 \mathrm{E}-40 \mathrm{R}-6,7-11$ & 519.47 & 28.30 & 54.37 \\
\hline-147 & 526. & 28. & 53. \\
\hline 7 & 5 & 28. & 54 \\
\hline 7 & & 28. & 54 \\
\hline & 2 & 28 & 55 \\
\hline $3,62-66$ & 5 & 28. & 53 \\
\hline$-45 R-3,124-127$ & 5 & 28 & 53.58 \\
\hline $4 \mathrm{R}-52-55$ & 570. & 28.5 & 52. \\
\hline (TD 08007 & 58 & 28 & 52. \\
\hline 605 & 4 & 28. & 52. \\
\hline$-49 R-2,97-101$ & 60 & 28. & 54. \\
\hline $792 \mathrm{E}-49 \mathrm{R}-4,90-94$ & 603.05 & 28.72 & 54.21 \\
\hline 792E-50R-3, $114-118$ & 611.54 & 28.74 & 53.95 \\
\hline $792 \mathrm{E}-52 \mathrm{R}-1,146-149$ & 628.26 & 28. & 53.88 \\
\hline $792 \mathrm{E}-54 \mathrm{R}-3,81-84$ & 649.91 & 28.86 & 55.41 \\
\hline $792 \mathrm{E}-55 \mathrm{R}-\mathrm{CC}, 7-11$ & 659.23 & $\begin{array}{l}28.80 \\
28.88\end{array}$ & $\begin{array}{l}53.41 \\
52.67\end{array}$ \\
\hline $792 \mathrm{E}-56 \mathrm{R}-1,36-39$ & 665.76 & 28.92 & 52.74 \\
\hline 792E-56R-5, 33-36 & 671.73 & 28.95 & 56.17 \\
\hline $792 \mathrm{E}-56 \mathrm{R}-6,108-111$ & 673.70 & 28.96 & 61.86 \\
\hline $792 \mathrm{E}-57 \mathrm{R}-3,102-105$ & $\begin{array}{l}013.10 \\
679.12\end{array}$ & $\begin{array}{l}28.96 \\
28.98\end{array}$ & $\begin{array}{l}6.86 \\
55.57\end{array}$ \\
\hline $792 \mathrm{E}-58 \mathrm{R}-4,8-12$ & 689.38 & 29.00 & 52.69 \\
\hline $792 \mathrm{E}-59 \mathrm{R}-3,44-48$ & 697.71 & 29.02 & 50.46 \\
\hline $792 \mathrm{E}-60 \mathrm{R}-4,127-130$ & 709.77 & 29.05 & 52.41 \\
\hline
\end{tabular}


Table 2 (continued).

\begin{tabular}{|c|c|c|c|c|c|c|c|}
\hline & & & & & & & \\
\hline interval $(\mathrm{cm})$ & (mbsf) & (Ma) & $\mathrm{SiO}_{2}$ & $\mathrm{TiO}_{2}$ & $\mathrm{Al}_{2} \mathrm{O}_{3}$ & $\mathrm{MnO}$ & $\mathrm{MgO}$ \\
\hline 126- (Cont.) & & & & & & & \\
\hline $792 \mathrm{E}-61 \mathrm{R}-2,32-36$ & 715.42 & 29.10 & 52.06 & 0.76 & 18.06 & 0.12 & 5.17 \\
\hline 792E-62R-5, 57-60 & 728.12 & 29.50 & 54.43 & 0.82 & 19.66 & 0.10 & 5.17 \\
\hline $792 \mathrm{E}-63 \mathrm{R}-1,130-134$ & 733.80 & 29.70 & 52.62 & 0.72 & 19.83 & 0.08 & 5.55 \\
\hline $792 \mathrm{E}-64 \mathrm{R}-2,146-149$ & 745.11 & 29.90 & 52.43 & 0.72 & 19.48 & 0.09 & 6.16 \\
\hline $792 \mathrm{E}-65 \mathrm{R}-1,111-116$ & 752.91 & 30.10 & 53.55 & 0.66 & 20.55 & 0.13 & 7.62 \\
\hline $792 \mathrm{E}-66 \mathrm{R}-4,148-152$ & 767.48 & 30.30 & 56.68 & 0.71 & 17.04 & 0.19 & 7.19 \\
\hline $792 \mathrm{E}-67 \mathrm{R}-3,98-102$ & 775.18 & 30.50 & 52.05 & 0.75 & 17.23 & 0.40 & 9.37 \\
\hline $792 \mathrm{E}-68 \mathrm{R}-1,64$ & 781.54 & 30.70 & 51.78 & 0.81 & 19.29 & 1.35 & 5.80 \\
\hline $792 \mathrm{E}-68 \mathrm{R}-2,6-10$ & 782.20 & 30.90 & 51.12 & 0.89 & 17.68 & 0.53 & 6.43 \\
\hline $793 \mathrm{~A}-6 \mathrm{H}-2,79-82$ & 44.29 & 0.40 & 66.50 & 0.60 & 13.68 & 0.14 & 1.59 \\
\hline $793 \mathrm{~A}-6 \mathrm{H}-3,4448$ & 45.44 & 0.42 & 62.45 & 0.64 & 14.07 & 0.14 & 2.40 \\
\hline $793 \mathrm{~A}-7 \mathrm{H}-1,94-98$ & 52.54 & 0.49 & 64.84 & 0.59 & 14.52 & 0.15 & 1.95 \\
\hline $793 \mathrm{~A}-7 \mathrm{H}-2,37-40$ & 53.47 & 0.50 & 58.12 & 0.73 & 15.76 & 0.17 & 2.86 \\
\hline $793 \mathrm{~A}-8 \mathrm{H}-1,32-36$ & 61.52 & 0.55 & 52.31 & 1.04 & 14.16 & 0.21 & 4.53 \\
\hline $793 \mathrm{~A}-9 \mathrm{H}-2,111-114$ & 73.51 & 0.70 & 52.62 & 1.14 & 14.41 & 0.22 & 3.96 \\
\hline $793 \mathrm{~A}-10 \mathrm{H}-2,65-68$ & 81.56 & 0.75 & 59.66 & 0.83 & 14.05 & 0.16 & 3.14 \\
\hline $793 \mathrm{~A}-11 \mathrm{H}-4,44-47$ & 95.04 & 0.80 & 68.92 & 0.49 & 13.61 & 0.14 & 1.29 \\
\hline $793 \mathrm{~B}-3 \mathrm{R}-1,66-70$ & 604.96 & 15.10 & 55.57 & 0.88 & 14.68 & 0.18 & 4.39 \\
\hline $793 \mathrm{~B}-9 \mathrm{R}-1,3-4$ & 662.13 & 15.90 & 67.64 & 0.68 & 13.53 & 0.15 & 1.52 \\
\hline 793B-10R-1, $108-111$ & 672.88 & 16.20 & 62.37 & 0.71 & 13.65 & 0.17 & 3.13 \\
\hline $793 \mathrm{~B}-10 \mathrm{R}-2,13-16$ & 673.43 & 16.22 & 68.87 & 0.51 & 12.87 & 0.12 & 1.78 \\
\hline $793 \mathrm{~B}-11 \mathrm{R}-1,104-107$ & 682.44 & 16.50 & 56.39 & 0.58 & 16.75 & 0.17 & 4.52 \\
\hline $793 \mathrm{~B}-14 \mathrm{R}-1,125-127$ & 711.65 & 17.10 & 68.49 & 0.60 & 13.48 & 0.15 & 1.48 \\
\hline $793 \mathrm{~B}-15 \mathrm{R}-1,85-88$ & 720.95 & 18.20 & 68.42 & 0.56 & 13.90 & 0.15 & $\begin{array}{l}1.59 \\
1.59\end{array}$ \\
\hline $793 \mathrm{~B}-19 \mathrm{R}-4,47-51$ & 763.02 & 23.50 & 53.19 & 0.83 & 19.51 & 0.88 & 4.72 \\
\hline $793 \mathrm{~B}-20 \mathrm{R}-4,91-95$ & 773.08 & 24.95 & 55.55 & 0.82 & 16.83 & 0.19 & 3.66 \\
\hline $793 \mathrm{~B}-2 \mathrm{IR}-2,109-112$ & 780.29 & 25.25 & 56.93 & 0.86 & 17.05 & 0.17 & 3.67 \\
\hline $793 \mathrm{~B}-2 \mathrm{IR}-3,25-28$ & 780.95 & 25.30 & 59.72 & 0.61 & 17.81 & 0.13 & 2.50 \\
\hline $793 \mathrm{~B}-22 \mathrm{R}-3,33-36$ & 790.57 & 25.75 & 53.04 & 0.63 & 19.25 & 0.22 & 4.48 \\
\hline $793 \mathrm{~B}-23 \mathrm{R}-4,9-12$ & 801.46 & 26.60 & 50.51 & 0.76 & 18.30 & 0.22 & 5.19 \\
\hline 793B-24R-2, 5-9 & 808.25 & 27.15 & 51.23 & 0.80 & 18.64 & 0.19 & 4.49 \\
\hline 793B-25R-1, 147-151 & 817.87 & 27.60 & 53.17 & 0.89 & 17.09 & 0.18 & 4.81 \\
\hline 793B-26R-5, 125-129 & 832.82 & 27.80 & 51.51 & 0.69 & 17.74 & 0.22 & 7.97 \\
\hline $793 \mathrm{~B}-27 \mathrm{R}-6,35-40$ & 842.90 & 27.97 & 53.51 & 0.77 & 17.88 & 0.19 & 5.68 \\
\hline $793 \mathrm{~B}-28 \mathrm{R}-6,33-37$ & 851.96 & 28.05 & 54.02 & 0.76 & 17.75 & 0.19 & 4.03 \\
\hline $793 \mathrm{~B}-29 \mathrm{R}-5,109-112$ & 861.09 & 28.15 & 53.05 & 0.68 & 18.32 & 0.19 & 4.61 \\
\hline $793 \mathrm{~B}-30 \mathrm{R}-3,25-29$ & 867.65 & 28.20 & 50.98 & 0.59 & 18.63 & 0.25 & 6.81 \\
\hline $793 \mathrm{~B}-31 \mathrm{R}-5,113-117$ & 880.35 & 28.35 & 57.65 & 0.62 & 17.49 & 0.15 & 2.92 \\
\hline $793 \mathrm{~B}-32 \mathrm{R}-1,39-43$ & 884.09 & 28.40 & 53.66 & 0.59 & 20.29 & 0.18 & 3.40 \\
\hline $793 \mathrm{~B}-33 \mathrm{R}-7,82-85$ & 902.41 & 28.60 & 58.98 & 0.56 & 17.13 & 0.16 & 3.13 \\
\hline $793 \mathrm{~B}-34 \mathrm{R}-2,44-47$ & 904.84 & 28.64 & 56.43 & 0.62 & 18.52 & 0.17 & 3.09 \\
\hline $793 \mathrm{~B}-35 \mathrm{R}-2,139-142$ & 915.37 & 28.72 & 52.07 & 0.68 & 20.56 & 0.17 & 3.68 \\
\hline 793B-36R-6, 103-107 & 930.25 & 28.85 & 53.90 & 0.66 & 18.26 & 0.18 & 3.78 \\
\hline 793B-37R-4, 35-39 & 936.71 & 28.90 & 59.38 & 0.61 & 16.70 & 0.14 & 3.03 \\
\hline $793 \mathrm{~B}-38 \mathrm{R}-2,65-69$ & 943.76 & 28.95 & 62.01 & 0.70 & 15.34 & 0.13 & 2.55 \\
\hline $793 \mathrm{~B}-39 \mathrm{R}-1,24-27$ & 951.54 & 29.00 & 58.78 & 0.61 & 17.22 & 0.16 & 3.27 \\
\hline $793 \mathrm{~B}-40 \mathrm{R}-7,0-3$ & 967.87 & 29.17 & 57.29 & 0.61 & 16.04 & 0.19 & 3.85 \\
\hline $793 \mathrm{~B}-4 \mathrm{IR}-5,113-117$ & 977.36 & 29.22 & 60.34 & 0.65 & 15.20 & 0.20 & 2.76 \\
\hline $793 \mathrm{~B}-42 \mathrm{R}-4,31-35$ & 984.86 & 29.27 & 58.94 & 0.64 & 15.08 & 0.19 & 3.35 \\
\hline 793B-43R-5, 63-68 & 995.57 & 29.29 & 60.64 & 0.51 & 15.45 & 0.19 & 3.53 \\
\hline 793B-44R-2, $63-68$ & 1001.41 & 29.30 & 61.02 & 0.56 & 15.14 & 0.17 & 3.05 \\
\hline $793 \mathrm{~B}-45 \mathrm{R}-1,38-42$ & 1009.48 & 29.34 & 59.54 & 0.61 & 15.00 & 0.19 & 3.80 \\
\hline $793 \mathrm{~B}-46 \mathrm{R}-6,108-113$ & 1026.15 & 29.40 & 59.40 & 0.66 & 14.61 & 0.16 & 3.46 \\
\hline $793 \mathrm{~B}-47 \mathrm{R}-4,25-28$ & 1032.29 & 29.42 & 57.94 & 0.69 & 14.60 & 0.18 & 3.54 \\
\hline $793 \mathrm{~B}-48 \mathrm{R}-3,58-60$ & 1041.18 & 29.47 & 56.10 & 0.58 & 15.64 & 0.20 & 4.70 \\
\hline $793 \mathrm{~B}-49 \mathrm{R}-4,30-34$ & 1051.12 & 29.50 & 66.35 & 0.73 & 13.54 & 0.17 & 2.13 \\
\hline $793 \mathrm{~B}-50 \mathrm{R}-1,80-83$ & 1057.80 & 29.52 & 53.50 & 0.53 & 15.92 & 0.19 & 7.25 \\
\hline $793 \mathrm{~B}-51 \mathrm{R}-5,6-9$ & 1071.70 & 29.60 & 52.35 & 0.52 & 14.82 & 0.25 & 10.32 \\
\hline 793B-52R-1,93-97 & 1077.33 & 29.63 & 53.48 & 0.56 & 14.80 & 0.19 & 8.16 \\
\hline $793 \mathrm{~B}-53 \mathrm{R}-3,145-149$ & 1090.42 & 29.67 & 55.23 & 0.51 & $\begin{array}{l}14.8 \\
16.4\end{array}$ & 0.26 & 7.36 \\
\hline $793 \mathrm{~B}-54 \mathrm{R}-3,103-107$ & 1098.57 & 29.69 & 53.34 & 0.47 & 15.49 & 0.18 & 7.22 \\
\hline $793 \mathrm{~B}-56 \mathrm{R}-3,26-30$ & 1118.26 & 29.73 & 54.64 & 0.48 & 16.76 & 0.16 & 5.67 \\
\hline$-57 R-2,139-143$ & 1127.59 & 29.77 & 53.40 & 0.49 & 16.5 & 0.18 & 6.45 \\
\hline$-6,22-26$ & 1141.05 & 29.80 & 53.33 & 0.45 & 17.21 & 0.23 & 7.2 \\
\hline $3-59 R-5,74-77$ & 1149.91 & 29.85 & 51.81 & 0.51 & 15.10 & 0.21 & 8.2 \\
\hline $793 \mathrm{~B}-60 \mathrm{R}-1,107-111$ & 1154.27 & 29.88 & 46.99 & 0.49 & 15.41 & 0.18 & 6.90 \\
\hline 793B-62R-1, 81-85 & 1173.41 & 29.93 & 63.96 & 0.52 & 14.38 & 0.15 & 2.96 \\
\hline 793B-63R-3, 15-19 & 1185.35 & 29.97 & 56.43 & 0.51 & 16.35 & 0.42 & 5.79 \\
\hline 793B-64R-6, $14-18$ & 1198.98 & 29.98 & 55.16 & 0.54 & 18.27 & 0.16 & 4.53 \\
\hline 793B-65R-6, 101-105 & 1209.85 & 30.00 & 58.08 & 0.72 & 16.7 & 0.13 & 3.35 \\
\hline $793 \mathrm{~B}-66 \mathrm{R}-6,98-101$ & 1219.27 & 30.03 & 58.39 & 0.53 & 17.59 & 0.10 & 3.00 \\
\hline $793 \mathrm{~B}-67 \mathrm{R}-1,56-60$ & 1221.36 & 30.04 & 53.85 & 0.58 & 17.73 & 0.18 & 5.01 \\
\hline $793 \mathrm{~B}-67 \mathrm{R}-5,56-60$ & 1227.17 & 30.06 & 57.24 & 0.59 & 17.05 & 0.10 & 3.60 \\
\hline 793B-68R-3,92-96 & 1234.32 & 30.08 & 57.57 & 0.57 & 16.84 & 0.13 & 3.70 \\
\hline $793 \mathrm{~B}-69 \mathrm{R}-2,145-148$ & 1243.09 & $30.10^{\circ}$ & 54.98 & 0.58 & 17.77 & 0.17 & 4.77 \\
\hline $793 B-70 R-2,0-4$ & 1251.30 & 30.14 & 60.54 & 0.40 & 17.15 & 0.10 & 2.31 \\
\hline $793 \mathrm{~B}-71 \mathrm{R}-1,126-129$ & 1260.66 & 30.17 & 59.30 & 0.81 & 15.33 & 0.12 & 2.14 \\
\hline $793 \mathrm{~B}-72 \mathrm{R}-2,0-5$ & 1270.20 & 30.20 & 57.92 & 0.55 & 18.73 & 0.17 & 3.72 \\
\hline $793 \mathrm{~B}-73 \mathrm{R}-2,0-4$ & 1279 & 30.27 & 56.03 & 0.67 & 18.45 & 0.18 & 3.95 \\
\hline $793 \mathrm{~B}-74 \mathrm{R}-3,112-116$ & 1291.95 & 30.30 & $\begin{array}{l}30.03 \\
58.28\end{array}$ & $\begin{array}{l}0.01 \\
0.61\end{array}$ & $\begin{array}{l}18.43 \\
18.49\end{array}$ & $\begin{array}{l}0.18 \\
0.18\end{array}$ & 3.12 \\
\hline $793 \mathrm{~B}-75 \mathrm{R}-7,31-35$ & 1306.94 & 30.35 & 59.10 & 0.60 & 17.73 & 0.16 & 3.17 \\
\hline 793B-76R-5, 77-81 & 1313.98 & 30.47 & 58. & 0.52 & 19.35 & 0.15 & 3.0 \\
\hline & & 30. & 56 & 0.6 & 17. & 0.17 & 3.6 \\
\hline & & & & 0. & & 0.10 & 2.0 \\
\hline & & & & 0. & & 0 . & 2. \\
\hline 79 & 1352 & 30 & 57 & 0. & 18 & 0.15 & 2. \\
\hline $793 \mathrm{~B}-81 \mathrm{R}-5,38-42$ & 1361.69 & 30.85 & 56.69 & 0.62 & 17.67 & 0.14 & 3.5 \\
\hline $793 \mathrm{~B}-82 \mathrm{R}-3,75-79$ & 1368.85 & 30.90 & 56.11 & 0.70 & 17.28 & 0.15 & 3.99 \\
\hline
\end{tabular}


Table 3. Volatile-free trace element data.

\begin{tabular}{|c|c|c|c|}
\hline & & & \\
\hline interval $(\mathrm{cm})$ & (mbsf) & (Ma) & Sc \\
\hline $126-$ & & & \\
\hline $787 \mathrm{~A}-1 \mathrm{~W}-1,26-32$ & 0.26 & 0.01 & 24 \\
\hline 787B-IR-1, 48-52 & 0.48 & 0.01 & 32 \\
\hline 787B-2R-1, 6-10 & 2.36 & 0.04 & 32 \\
\hline $787 \mathrm{~A}-1 \mathrm{~W}-3,15-21$ & 3.15 & 0.05 & 29 \\
\hline $787 \mathrm{~B}-2 \mathrm{R}-1,103-107$ & 3.33 & 0.06 & 34 \\
\hline $787 \mathrm{~B}-3 \mathrm{R}-2,76-79$ & 14.16 & 0.25 & 32 \\
\hline 787B-7R-1, $3-7$ & 49.83 & 24.20 & 34 \\
\hline $787 \mathrm{~B}-9 \mathrm{R}-2,47-50$ & 70.97 & 25.00 & 30 \\
\hline $787 \mathrm{~B}-14 \mathrm{R}-2,69-73$ & 119.69 & 26.70 & 43 \\
\hline $87 \mathrm{~B}-14 \mathrm{R}-3,72-76$ & 120.93 & 26.80 & 40 \\
\hline $787 \mathrm{~B}-15 \mathrm{R}-1,108-111$ & 128.28 & 27.10 & 40 \\
\hline 7871 & 131.91 & 27.18 & 38 \\
\hline 78 & 137.26 & 27.25 & 41 \\
\hline 787. & 152.71 & 27.40 & 36 \\
\hline $18 \mathrm{R}-1,17-20$ & 156.37 & 27.50 & 43 \\
\hline $787 \mathrm{~B}-18 \mathrm{R}-3,142-145$ & 160.02 & 27.55 & 41 \\
\hline 787B-20R-1, 15-16 & 175.65 & 27.80 & 34 \\
\hline $787 \mathrm{~B}-20 \mathrm{R}-4,20-23$ & 180.20 & 27.85 & 34 \\
\hline 787B-21R-1, 65-69 & 185.75 & 27.87 & 35 \\
\hline 787B-22R-1, 47-50 & 195.27 & 27.90 & 38 \\
\hline $787 \mathrm{~B}-23 \mathrm{R}-4,18-21$ & 209.08 & 28.00 & 31 \\
\hline $787 \mathrm{~B}-24 \mathrm{R}-6,91-94$ & 222.51 & 28.08 & 21 \\
\hline $787 \mathrm{~B}-25 \mathrm{R}-1,119-122$ & 224.89 & 28.10 & 13 \\
\hline 787B-25R-4, $10-13$ & 228.30 & 28.12 & 48 \\
\hline 787B-26R-5, $19-22$ & 239.59 & 28.28 & 30 \\
\hline 787B-27R-1,77-80 & 243.77 & 28.30 & 33 \\
\hline $787 \mathrm{~B}-27 \mathrm{R}-\mathrm{CC}, 0-3$ & 248.12 & 28.38 & 31 \\
\hline $3,62-65$ & 275.62 & 28.58 & 13 \\
\hline 787 & 283.05 & 28.63 & 25 \\
\hline 78 & 291.8 & 28.7 & 28 \\
\hline 78 & 302.16 & 28.8 & 29 \\
\hline 78 & 13.36 & 0.10 & 20 \\
\hline $788 \mathrm{C}-3 \mathrm{H}-1,42-46$ & 20.62 & 0.15 & 23 \\
\hline $788 \mathrm{~A}-4 \mathrm{R}-1,18-21$ & 26.38 & 0.20 & 19 \\
\hline $788 \mathrm{C}-4 \mathrm{H}-1,64-67$ & 30.34 & 2.35 & 92 \\
\hline $8 \mathrm{C}-4 \mathrm{H}-4,46-49$ & 34.66 & 2.36 & 22 \\
\hline $788 \mathrm{C}-8 \mathrm{H}-1,20-23$ & 67.90 & 2.64 & 22 \\
\hline $788 \mathrm{C}-8 \mathrm{H}-2,107-110$ & 70.27 & 2.68 & 22 \\
\hline $788 \mathrm{C}-9 \mathrm{H}-1,85-88$ & 78.05 & 2.71 & 24 \\
\hline $788 \mathrm{C}-11 \mathrm{H}-\mathrm{CC}, 13-16$ & 102.53 & 2.89 & 18 \\
\hline $788 \mathrm{C}-13 \mathrm{H}-5,120-124$ & 122.40 & 2.98 & 15 \\
\hline $57-60$ & 131.27 & 3.10 & 15 \\
\hline $5 \rightarrow 9$ & 134.25 & 3.12 & 13 \\
\hline $7-1$ & 143.7 & 3.19 & 20 \\
\hline 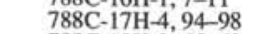 & $\begin{array}{l}158.6 \\
158.6\end{array}$ & 3.28 & 18 \\
\hline 78 & 164.8 & 3.31 & 12 \\
\hline & 172.8 & 3.38 & 21 \\
\hline & 183.6 & 3.4 & 23 \\
\hline $40-4$ & 19 & 3.5 & 21 \\
\hline & 21 & 3.6 & 22 \\
\hline $110-114$ & 222 . & 3.70 & 20 \\
\hline $1,24-27$ & 268.1 & 4.14 & 46 \\
\hline$C, 7-10$ & 279. & 4.17 & 41 \\
\hline $1,29-32$ & 287. & 4.20 & 38 \\
\hline $9 \mathrm{R}-1,101-105$ & 297. & 4.23 & 39 \\
\hline$-1 \mathrm{H}-5,132-1$ & 7 & 0.01 & 17 \\
\hline 01 & 14.8 & 0. & 28 \\
\hline $\mathrm{H}-2,21-24$ & & 0. & 25 \\
\hline 09 & & 0. & 22 \\
\hline & 31.2 & 0. & 17 \\
\hline & 31 & 0. & 14 \\
\hline & 34. & 0. & 23 \\
\hline $0 \mathrm{H}$ & 47.1 & 0.0 & 13 \\
\hline & 60.3 & 0. & 16 \\
\hline & & 0. & 34 \\
\hline & & 0. & it \\
\hline 7 & & 0. & 2 \\
\hline & & 0. & 19 \\
\hline 07 & & 0. & 11 \\
\hline & & & 13 \\
\hline 55 & & 0 . & 15 \\
\hline & & & 22 \\
\hline & & 0 . & 24 \\
\hline & & & $\tau$ \\
\hline $2 \mathrm{X}$ & & 0. & 20 \\
\hline & 2 & 0.43 & 20 \\
\hline $5=160$ & 22 & 0.55 & 24 \\
\hline 4 & 266.52 & 0.9 & 24 \\
\hline $1 \mathrm{~A}-3 \mathrm{H}-1,83-86$ & & 0.8 & 17 \\
\hline $142-145$ & & 0. & 27 \\
\hline & 35.4 & 0. & 27 \\
\hline & 410 & 0. & 18 \\
\hline & & & 11 \\
\hline & & & 1 \\
\hline & & & \\
\hline & & & \\
\hline & & & \\
\hline & & & 17 \\
\hline & & 0. & 13 \\
\hline & & 0.1 & 30 \\
\hline $791 \mathrm{~A}-34 \mathrm{X}-\mathrm{CC}, 12-1$ & 304.9 & 0.1 & 11 \\
\hline $791 \mathrm{~A}-41 \mathrm{X}-\mathrm{CC}, 1-7$ & 371.71 & 0.19 & 14 \\
\hline
\end{tabular}


Table 3 (continued).

\begin{tabular}{|c|c|c|c|}
\hline & & & \\
\hline interval $(\mathrm{cm})$ & (mbsf) & (Ma) & Sc \\
\hline 126- (Cont.) & & & \\
\hline $791 \mathrm{~A}-46 \mathrm{X}-\mathrm{CC}, 8-13$ & 418.88 & 0.22 & \\
\hline $791 \mathrm{~B}-11 \mathrm{R}-\mathrm{CC}, 13-16$ & 483.03 & 0.25 & 18 \\
\hline $791 \mathrm{~B}-15 \mathrm{R}-1,3-6$ & 521.53 & 0.29 & 16 \\
\hline $791 \mathrm{~B}-15 \mathrm{R}-\mathrm{CC}, 3-$ & 521.63 & 0.29 & 16 \\
\hline 1,21 & 800.91 & 0.86 & 26 \\
\hline 104 & 801.71 & 0.87 & 25 \\
\hline 7 & 812.36 & 0.92 & 36 \\
\hline $\mathrm{CC}, 1$ & 813.57 & 0.92 & 26 \\
\hline, $72-$ & 820.72 & 0.94 & 19 \\
\hline$-1,36-39$ & 829.96 & 0.96 & 32 \\
\hline $1 \mathrm{H}-6,52-55$ & 8.02 & 0.12 & 23 \\
\hline $2 \mathrm{~A}-1 \mathrm{H}-6,95-99$ & 8.45 & 0.13 & 33 \\
\hline $792 \mathrm{~A}-2 \mathrm{H}-1,87-90$ & 10.57 & 0.14 & 34 \\
\hline $2 \mathrm{~A}-2 \mathrm{H}-4,46-50$ & 14.66 & 0.19 & 21 \\
\hline $792 \mathrm{~A}-3 \mathrm{H}-5,17-20$ & 25.27 & 0.32 & 29 \\
\hline $792 \mathrm{~A}-4 \mathrm{H}-5,14-17$ & 34.54 & 0.44 & 39 \\
\hline $792 \mathrm{~A}-5 \mathrm{H}-2,52-55$ & 39.82 & 0.50 & 17 \\
\hline $792 \mathrm{~A}-6 \mathrm{H}-4,82-85$ & 52.52 & 0.65 & 37 \\
\hline $792 \mathrm{~B}-2 \mathrm{H}-3,145-148$ & 54.45 & 0.68 & 28 \\
\hline $792 \mathrm{~B}-2 \mathrm{H}-6,132-135$ & 58.41 & 0.75 & 37 \\
\hline $792 \mathrm{~B}-3 \mathrm{H}-3,26-30$ & 62.86 & 0.77 & 41 \\
\hline $118-$ & 63.45 & 0.78 & 43 \\
\hline 7 & 65 & 0.8 & 43 \\
\hline & 68 & 0.8 & 29 \\
\hline 7 & 77.42 & 0.90 & 31 \\
\hline $\mathrm{C}, 2$ & 79.05 & 0.91 & 25 \\
\hline $792 \mathrm{~A}-10$ & 89.34 & 1.00 & 21 \\
\hline 792B-8X-3, 62-65 & 111.52 & 2.39 & 28 \\
\hline 792E-1R-1, 92-96 & 136.52 & 2.70 & 33 \\
\hline 792E-4R-2, 54-57 & 166.54 & 3.20 & 41 \\
\hline $792 \mathrm{E}-8 \mathrm{R}-1,18-21$ & 203.28 & 6.20 & 40 \\
\hline 792E-9R-3, 68-73 & 216.28 & 6.60 & 46 \\
\hline $792 \mathrm{E}-9 \mathrm{R}-5,26-31$ & 217.86 & 6.70 & 41 \\
\hline $792 \mathrm{E}-11 \mathrm{R}-1,87-90$ & 232.77 & 6.90 & 41 \\
\hline $792 \mathrm{E}-12 \mathrm{R}-3,108-110$ & 245.58 & 7.40 & 34 \\
\hline $13 R-2,2$ & 252.95 & 7.50 & 39 \\
\hline C, 1 & 261.09 & 7.70 & 39 \\
\hline $93-$ & 282 & 9.50 & 36 \\
\hline 46 & 291.86 & 10.00 & 35 \\
\hline 7 & 293.12 & 10.10 & 36 \\
\hline & 301.43 & 10.5 & 34 \\
\hline & 322.15 & 11. & 33 \\
\hline 21 & 348.78 & 12.7 & 54 \\
\hline $2, y,-9$ & 398.44 & 22.6 & 33 \\
\hline E-30R-1, $116-119$ & 416.36 & 24.9 & 33 \\
\hline E-31R-2, 19-22 & 426.59 & 25. & 25 \\
\hline $2 \mathrm{E}-32 \mathrm{R}-3,81-84$ & 438.41 & 26. & 28 \\
\hline 792E-33R-1, $105-108$ & 445.25 & 2 & 37 \\
\hline$-C C, 20-2$ & 458.86 & 2 & 34 \\
\hline $2 \mathrm{E}-36 \mathrm{R}-1,30-$ & 473.50 & 28. & 41 \\
\hline .57 & 4 & & 41 \\
\hline 79 & 4 & & 34 \\
\hline 1.28 & 5 & & 31 \\
\hline 257 & 5 & & 38 \\
\hline 79 & 51 & & 28 \\
\hline 79 & 526. & 28. & 35 \\
\hline 75 & 531.95 & 28. & 30 \\
\hline & 539.50 & 28. & 35 \\
\hline & 5 & 2 & 24 \\
\hline & 55 & 28 & 28 \\
\hline & & 2 & 33 \\
\hline & & 2 & 29 \\
\hline $88-$ & & & 39 \\
\hline 7 & & & 30 \\
\hline 7 & & & 2 \\
\hline 7 & & & 27 \\
\hline 18 & & & 34 \\
\hline & & & 34 \\
\hline & & & 2 \\
\hline & & & 4 \\
\hline & & & 40 \\
\hline & & & \\
\hline & 6 & & \\
\hline & 6 & & 2 \\
\hline $792 \mathrm{E}-$ & 689 & 29. & 43 \\
\hline & 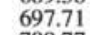 & & 52 \\
\hline & & & 3 \\
\hline & & & 4 \\
\hline & & & 2 \\
\hline & & & \\
\hline & & & \\
\hline & & & \\
\hline & & & \\
\hline & & & 3 \\
\hline & 78 & & 41 \\
\hline & & 30. & 45 \\
\hline & & 0. & 19 \\
\hline & 45 & 0. & 22 \\
\hline & 52. & 0. & 25 \\
\hline $\mathrm{H}-2,37$ & 53.47 & 0. & 34 \\
\hline $793 \mathrm{~A}$ & 61.52 & 0. & 57 \\
\hline $793 \mathrm{~A}-9 \mathrm{H}-2,111-114$ & 73.51 & 0.70 & 41 \\
\hline
\end{tabular}


Table 3 (continued).

\begin{tabular}{|c|c|c|c|c|c|c|c|c|c|c|c|c|c|c|c|c|c|}
\hline \multirow{2}{*}{$\begin{array}{l}\text { Core, section, } \\
\text { interval }(\mathrm{cm})\end{array}$} & \multirow{2}{*}{$\begin{array}{l}\text { Depth } \\
\text { (mbsf) }\end{array}$} & \multirow{2}{*}{$\begin{array}{l}\text { Age } \\
\text { (Ma) }\end{array}$} & \multicolumn{15}{|c|}{ Trace elements (ppm) } \\
\hline & & & Sc & $\mathrm{Ti}$ & $\mathrm{V}$ & $\mathrm{Cr}$ & $\mathrm{Ni}$ & $\mathrm{Cu}$ & $\mathrm{Zn}$ & $\mathbf{G a}$ & $\mathbf{R b}$ & $\mathrm{Sr}$ & $\mathrm{Y}$ & $\mathrm{Zr}$ & $\mathrm{Ba}$ & $\mathrm{Ce}$ & $\mathrm{Pb}$ \\
\hline 126- (Cont.) & & & & & & & & & & & & & & & & & \\
\hline $793 \mathrm{~A}-10 \mathrm{H}-2,65-68$ & 81.56 & 0.75 & 34 & 5193 & 292 & 23 & 10 & 66 & 120 & 15 & 8 & 191 & 29 & 75 & 293 & 27 & 7 \\
\hline $793 \mathrm{~A}-11 \mathrm{H}-4,44-47$ & 95.04 & 0.80 & 20 & 3236 & 60 & $<5$ & $<5$ & 18 & 93 & 14 & 12 & 208 & 40 & 96 & 512 & 27 & \\
\hline $793 \mathrm{~B}-3 \mathrm{R}-1,66-70$ & 604.96 & 15.10 & 46 & 6793 & 522 & 15 & 16 & 226 & 133 & $\begin{array}{l}14 \\
16\end{array}$ & 12 & $\begin{array}{l}168 \\
168\end{array}$ & 23 & 40 & 241 & $<20$ & $<5$ \\
\hline $793 \mathrm{~B}-10 \mathrm{R}-1,108-111$ & 672.88 & 16.20 & 32 & 4862 & 248 & 6 & $\begin{array}{r}10 \\
6\end{array}$ & $\begin{array}{r}220 \\
66\end{array}$ & 114 & 15 & 12 & 169 & 30 & 60 & 191 & 27 & $<5$ \\
\hline $793 \mathrm{~B}-11 \mathrm{R}-1,104-107$ & 682.44 & 16.50 & 32 & $\begin{array}{l}4802 \\
4282\end{array}$ & 323 & 49 & $\begin{array}{r}0 \\
16\end{array}$ & $\begin{array}{l}60 \\
78\end{array}$ & $\begin{array}{l}114 \\
106\end{array}$ & 16 & 12 & $\begin{array}{l}179 \\
178-29\end{array}$ & $\begin{array}{l}30 \\
18\end{array}$ & 30 & 115 & 28 & 5 \\
\hline 93B-19R-4, 47-51 & 763.02 & 23.50 & 39 & 5733 & 366 & $\begin{array}{r}49 \\
6\end{array}$ & 10 & 86 & $\begin{array}{r}100 \\
92\end{array}$ & 20 & 23 & 238 & 29 & 72 & 316 & 48 & $<5$ \\
\hline $793 \mathrm{~B}-20 \mathrm{R}-4,91-95$ & 773.08 & 24.95 & 29 & 4892 & 323 & $<5$ & 9 & 80 & 94 & 18 & 12 & 877 & 22 & 73 & 411 & 39 & $<5$ \\
\hline $793 \mathrm{~B}-2 \mathrm{IR}-2,109-112$ & 780.29 & 25.25 & 34 & 5242 & 239 & $<5$ & 6 & 98 & 93 & 18 & 15 & 488 & 40 & 102 & 400 & 26 & 5 \\
\hline $1 R-3,25-28$ & 780.95 & 25.30 & 21 & 4169 & 223 & $<5$ & $<5$ & 51 & 75 & 12 & 12 & 1350 & 19 & & 889 & 43 & 12 \\
\hline $33-36$ & 790.57 & 25.75 & 29 & 4762 & 359 & 6 & 10 & 112 & 86 & 14 & 18 & 22 & 18 & 51 & 541 & 49 & \\
\hline 79 & 801.4 & 26.60 & 35 & 5378 & 452 & $<5$ & 11 & 148 & 94 & 17 & 3 & 34 & 19 & 0 & 71 & 25 & 0 \\
\hline$-2,5$ & 808.25 & 27.15 & 33 & 5208 & 403 & 8 & $<5$ & 151 & 82 & 17 & 6 & 396 & 23 & 59 & 217 & 22 & $<5$ \\
\hline-151 & 817.87 & 27.60 & 36 & 5910 & 459 & 33 & 11 & 143 & 106 & 10 & 10 & 371 & 19 & 54 & 495 & 24 & $<5$ \\
\hline $5,125-129$ & 832.82 & 27.80 & 36 & 5305 & 430 & 31 & $2 i$ & 133 & 90 & 16 & 14 & 91 & 20 & 43 & 216 & 32 & $<$ \\
\hline$-27 \mathrm{R}-6,35-40$ & 842.90 & 27.97 & 38 & 5368 & 386 & 43 & 15 & 131 & 85 & 19 & 6 & 149 & 20 & 51 & 123 & 45 & 5 \\
\hline 793B-28R-6, 33-37 & 851.96 & 28.05 & 34 & 5547 & 365 & 9 & 8 & 117 & 92 & 16 & 19 & 225 & 28 & 70 & 597 & 53 & $<5$ \\
\hline $793 \mathrm{~B}-29 \mathrm{R}-5,109-112$ & 861.09 & 28.15 & 39 & 5570 & 411 & $<5$ & 5 & 144 & 108 & 19 & 17 & 188 & 19 & 55 & 385 & 62 & 10 \\
\hline $793 \mathrm{~B}-30 \mathrm{R}-3,25-29$ & 867.65 & 28.20 & 35 & 5436 & 437 & $<5$ & 13 & 143 & 85 & 20 & 22 & 118 & 20 & 44 & 223 & 43 & $<5$ \\
\hline $793 \mathrm{~B}-31 \mathrm{R}-5,113-117$ & 880.35 & 28.35 & 28 & 4533 & 257 & $<5$ & $<5$ & 113 & 101 & 16 & 15 & 655 & 26 & 76 & 532 & 29 & $<5$ \\
\hline $793 \mathrm{~B}-32 \mathrm{R}-1,39-43$ & 884.09 & 28.40 & 27 & 4835 & 248 & $<5$ & $<5$ & 90 & 86 & 20 & 10 & 540 & 21 & 54 & 491 & 30 & $<5$ \\
\hline $793 \mathrm{~B}-33 \mathrm{R}-7,82-85$ & 902.41 & 28.60 & 23 & 4066 & 203 & 6 & 7 & 74 & 86 & 17 & 10 & 405 & 21 & 66 & 337 & 32 & -5 \\
\hline $793 \mathrm{~B}-34 \mathrm{R}-2,44-47$ & 904.84 & 28.64 & 24 & 4536 & 244 & $<5$ & $<5$ & 90 & 98 & 15 & 9 & 457 & 22 & 60 & 504 & 44 & \\
\hline $793 \mathrm{~B}-35 \mathrm{R}-2,139-142$ & 915.37 & 28.72 & 27 & $\begin{array}{l}4350 \\
4776\end{array}$ & 305 & $<5$ & $<$ & 90 & $\begin{array}{l}78 \\
79\end{array}$ & 22 & 7 & 252 & 18 & 37 & 383 & 28 & $<5$ \\
\hline 793B-36R-6, 103-107 & 930.25 & 28.85 & 24 & $\begin{array}{l}4710 \\
4964\end{array}$ & 270 & 32 & 10 & $\begin{array}{l}90 \\
73\end{array}$ & 83 & 15 & 6 & 246 & 2 & 59 & 307 & 21 & 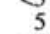 \\
\hline $37 \mathrm{R}-4,35-39$ & 936.71 & 28.90 & $\begin{array}{l}24 \\
18\end{array}$ & $\begin{array}{l}4904 \\
3566\end{array}$ & 201 & $<5$ & $<5$ & $\begin{array}{l}13 \\
66\end{array}$ & $\begin{array}{l}83 \\
70\end{array}$ & 15 & 10 & $\begin{array}{l}240 \\
387\end{array}$ & 24 & 61 & 541 & 41 & $<$ \\
\hline$R-2,65$ & 943.76 & 28.95 & 25 & $\begin{array}{l}3500 \\
4563\end{array}$ & 275 & $<$ & ${ }_{6}^{\infty}$ & $\begin{array}{l}60 \\
82\end{array}$ & 114 & 13 & $\begin{array}{r}10 \\
7\end{array}$ & 273 & 22 & $\begin{array}{l}01 \\
59\end{array}$ & 378 & $\begin{array}{l}41 \\
22\end{array}$ & 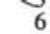 \\
\hline $39 R-1,24-27$ & 951.54 & 29.00 & 24 & $\begin{array}{l}4303 \\
4635\end{array}$ & 237 & $<5$ & $\begin{array}{l}0 \\
6\end{array}$ & $\begin{array}{l}82 \\
74\end{array}$ & $\begin{array}{r}114 \\
82\end{array}$ & 18 & 9 & 257 & 32 & 93 & $\begin{array}{r}318 \\
200\end{array}$ & 49 & 7 \\
\hline $70-3$ & 967.87 & 29.17 & 30 & 5313 & 325 & 26 & 5 & 83 & 99 & 14 & 17 & 280 & 28 & 63 & 219 & 42 & 6 \\
\hline 117 & 977.36 & 29.22 & 30 & 5709 & 263 & $<5$ & $<5$ & 71 & 110 & 19 & 19 & 23 & & 73 & 22 & 6 & 6 \\
\hline & 984.86 & 29.27 & 31 & 5430 & 279 & $<5$ & 5 & 73 & 100 & 17 & 18 & 25 & 3 & 74 & 216 & $<20$ & 6 \\
\hline 88 & 995.57 & 29.29 & 25 & 4833 & 250 & 7 & 7 & 88 & 92 & 16 & 13 & 268 & 29 & 77 & 265 & 33 & 5 \\
\hline B-44R-2, $63-68$ & 1001.41 & 29.30 & 28 & 5408 & 251 & $<5$ & $<5$ & 50 & 113 & 16 & 10 & 308 & 29 & 68 & 234 & 52 & $\delta$ \\
\hline$-45 R-1,38-42$ & 1009.48 & 29.34 & 36 & 5593 & 349 & $<5$ & 6 & 129 & 104 & 18 & 12 & 267 & 30 & 75 & 190 & 30 & 9 \\
\hline$-46 \mathrm{R}-6,108-113$ & 1026.15 & 29.40 & 30 & 5639 & 393 & $<$ & $<$ & 146 & 111 & 17 & 19 & 294 & 27 & 59 & 192 & 46 & 7 \\
\hline $793 \mathrm{~B}-47 \mathrm{R}-4,25-28$ & 1032.29 & 29.42 & 33 & 6226 & 437 & $<5$ & $<5$ & is6 & 107 & is & 24 & 241 & 32 & 66 & 153 & 22 & 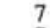 \\
\hline $793 \mathrm{~B}-48 \mathrm{R}-3,58-60$ & 1041.18 & 29.47 & 37 & 5356 & 360 & 31 & 10 & 118 & 100 & 16 & 10 & 268 & 27 & 59 & 148 & 43 & 7 \\
\hline$-4,30-34$ & 1051.12 & 29.50 & 20 & 6757 & 69 & $<5$ & $<5$ & 21 & 120 & 18 & 4 & 268 & 47 & 138 & 312 & 40 & 10 \\
\hline$-1,80-83$ & 1057.80 & 29.52 & 44 & 4523 & 388 & 145 & 62 & 98 & 78 & 12 & 21 & 215 & 15 & 35 & 129 & $<20$ & 5 \\
\hline 5,6 & 1071.70 & 29.60 & 38 & 5261 & $\begin{array}{l}368 \\
447\end{array}$ & 77 & $\begin{array}{l}02 \\
26\end{array}$ & 115 & 9 & 16 & 4 & 228 & 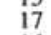 & 45 & 57 & 36 & 7 \\
\hline , $33-9$ & 10 & 29.63 & 39 & 4292 & 360 & 161 & 56 & 90 & 9 & 14 & 11 & 3. & 1 & 34 & 58 & 42 & $<$ \\
\hline 145 & 10 & 29.67 & 35 & $\begin{array}{l}4292 \\
4693\end{array}$ & $\begin{array}{l}300 \\
411\end{array}$ & $\begin{array}{r}101 \\
92\end{array}$ & 32 & $\begin{array}{r}90 \\
132\end{array}$ & 9 & 14 & 4 & 156 & 1 & 7 & 161 & 32 & 5 \\
\hline 10 & is & 29 & 41 & $\begin{array}{l}4093 \\
4277\end{array}$ & $\begin{array}{l}411 \\
396\end{array}$ & $\begin{array}{r}92 \\
147\end{array}$ & 41 & 126 & 8 & $\begin{array}{l}14 \\
16\end{array}$ & 4 & 2 & 1 & 34 & 52 & $<20$ & 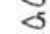 \\
\hline 79 & 1118. & $\begin{array}{l}29.09 \\
29.73\end{array}$ & $\begin{array}{l}41 \\
30\end{array}$ & 4292 & $\begin{array}{l}390 \\
377\end{array}$ & $\begin{array}{r}147 \\
40\end{array}$ & $\begin{array}{l}41 \\
18\end{array}$ & $\begin{array}{l}120 \\
100\end{array}$ & 8 & 14 & $\begin{array}{l}4 \\
4\end{array}$ & 567 & 1 & 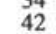 & 173 & 26 & $<5$ \\
\hline $139-143$ & 1127. & 29.77 & 32 & 4717 & 355 & 79 & 31 & 117 & 85 & 16 & 7 & 179 & 2 & 3 & 93 & 40 & $\delta$ \\
\hline & 1141. & 29. & 41 & 4788 & 38 & 71 & 30 & 80 & (1) & 15 & 9 & 3 & & & & 76 & 7 \\
\hline & & & 39 & 44 & 42 & 86 & 27 & 10 & 8 & 15 & 4 & 56 & 1 & & 23 & & $\delta$ \\
\hline-111 & 11 & 2 & 36 & & 40 & 85 & 31 & 9 & 7 & 13 & 9 & 16 & 16 & & 2 & & $<5$ \\
\hline & & & 21 & 47 & 10 & $<5$ & $<5$ & 27 & 10 & 18 & 4 & 4 & 4 & 12 & 26 & 48 & $<5$ \\
\hline $15-19$ & & & 32 & 35 & 25 & 38 & 18 & 75 & 10 & 13 & 8 & 4 & & & & & $<5$ \\
\hline & & & 24 & & 28 & 22 & 13 & 140 & 8 & 18 & 5 & 28 & & & 6 & $<20$ & $<5$ \\
\hline 05 & & & 29 & & 35 & 9 & $<5$ & 89 & 9 & 18 & 6 & 382 & 18 & 5 & 125 & 45 & $<5$ \\
\hline & & & 20 & & 25 & 10 & 5 & 96 & 7 & 15 & 13 & 217 & 18 & & 182 & 27 & 5 \\
\hline & 12 & & 28 & 48 & 36 & 19 & 8 & 120 & 8 & & 8 & & 1 & & & 38 & $<5$ \\
\hline 7 & 12 & & 29 & 45 & 27 & 10 & $\sum_{5}^{\circ}$ & 122 & 6 & 5 & 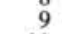 & & & & 105 & 39 & $<$ \\
\hline 7 & 12 & & 27 & 39 & 25 & 16 & 7 & $\begin{array}{r}69 \\
\end{array}$ & 82 & 16 & 11 & 217 & 17 & 5 & 141 & $<20$ & $<5$ \\
\hline 7 & 1243. & & 21 & 39 & 26 & $<5$ & 7 & 117 & $\begin{array}{l}62 \\
69\end{array}$ & $\begin{array}{l}10 \\
13\end{array}$ & 8 & & 21 & & 173 & 39 & $<5$ \\
\hline 7 & 1251. & 30 & 20 & 3797 & 17 & $<5$ & $\frac{1}{5}$ & 61 & 76 & 14 & 12 & & 26 & & 201 & 65 & s \\
\hline-129 & 12 & 30. & 20 & 5344 & $\begin{array}{l}170 \\
389\end{array}$ & 7 & $<5$ & 200 & 82 & $\begin{array}{l}14 \\
13\end{array}$ & 11 & & 2 & & 263 & 36 & 5 \\
\hline 79 & 1270.2 & 30. & 28 & 4479 & $\begin{array}{l}389 \\
257\end{array}$ & 5 & 8 & $\begin{array}{r}200 \\
66\end{array}$ & $\begin{array}{l}8 \\
8 \\
\end{array}$ & 18 & 6 & $\begin{array}{l}302 \\
379\end{array}$ & 24 & & 316 & 36 & $<5$ \\
\hline $0-4$ & 1279. & 30. & 25 & 50 & 310 & $<5$ & $<5$ & 87 & 10 & 18 & 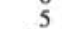 & 382 & 25 & & 18 & 57 & $<5$ \\
\hline & 17 & 30. & 29 & & 21 & $<5$ & $<$ & 51 & 10 & 17 & 15 & & 25 & & 16 & 43 & $<5$ \\
\hline & & 30. & 23 & 46 & 22 & $<$ & $<$ & & 8 & 20 & 7 & & 2 & & is & 38 & $<5$ \\
\hline & & & 24 & 38 & 18 r & $<$ & $<$ & & 9 & & 8 & & 2 & & & 12 & 6 \\
\hline & & & 2 & & & & & & & & 7 & & & & & & \\
\hline & & & $i$ & & & & & & & & 9 & & & & & & \\
\hline & & & i & & & $<$ & & & & & & & & & & & \\
\hline & & & 2 & & 22 & & $<$ & 5 & 8 & 2 & 0 & & & & & & \\
\hline & & & 3. & & 20 & 7 & 7 & 56 & 93 & 18 & 6 & 317 & & 8 & & & 5 \\
\hline $793 \mathrm{~B}-82 \mathrm{R}-3,75-79$ & 1368.85 & 30.90 & 27 & 5167 & 248 & $<$ & 7 & 56 & 97 & 18 & 9 & 321 & 28 & 88 & 122 & 28 & 5 \\
\hline
\end{tabular}

\section{TRACE ELEMENTS}

Not surprisingly, trace element data (Table 3 ) constrain the source terrane for the volcaniclastic sands and sandstones to a supra-subduction zone volcanic arc. Excluding rare Oligocene exceptions, Ti concentrations are less than the $7600 \mathrm{ppm}$ (about $1.3 \% \mathrm{TiO}_{2}$ ) average for normal mid-ocean-ridge basalt (N-MORB) (Fig. 8); such values are a signature of island-arc magmas. The Ti concentrations are higher than those typical of boninites (Hickey and Frey, 1982; Murton, 1989; Hickey-Vargas, 1989), even in the Oligocene sedimentary rocks lying above boninitic breccias ( $\mathrm{R}$. Taylor et al., this volume).

Considering only 109 samples with the composition of basalt and basic andesite, the $\mathrm{Ti} / \mathrm{V}$ ratio of 10-20 is typical of volcanic arcs (Fig. 9; Shervais, 1982). On discriminant diagrams of Pearce and
Cann (1973) and Pearce and Norry (1979), the samples plot mainly in the fields of island-arc tholeiites and calc-alkaline lavas (Figs. 10 and 11). Several samples with ages in the range 27-29 Ma are anomalous in that they have fairly low $\mathrm{Y}$ and $\mathrm{Sr}$ contents, so that they either plot in the fields of ocean-floor basalts, within-plate basalts, or outside of all fields.

The abundances of $\mathrm{Zr}$ and $\mathrm{Y}$, elements that exhibit low mobility during alteration, covary with $\mathrm{MgO}$ (Fig. 12). The $\mathrm{Zr} / \mathrm{Y}$ ratio decreases marginally up the stratigraphic section (Fig. 13); exceptionally high values of $8-10$ only occur in anomalously Y-poor samples near the base of the succession.

The samples analyzed by ICP-MS were selected using the following criteria: $\mathrm{SiO}_{2}$ content of $52 \%-58 \%$, preferably on the low side; low content of alkalis, particularly $\mathrm{K}_{2} \mathrm{O}$; higher than average $\mathrm{MgO}$ 


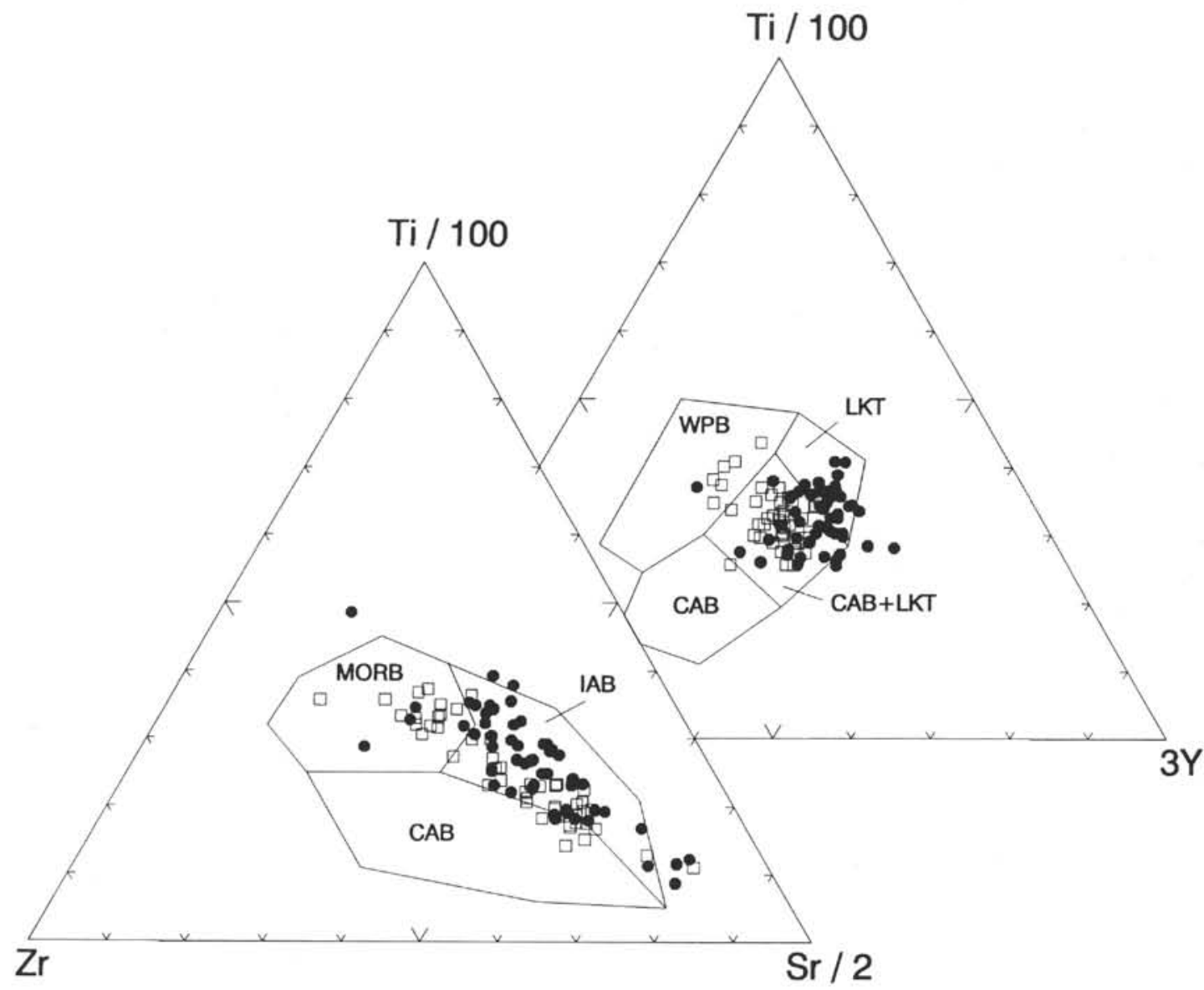

Figure 10. Petrological discrimination diagrams (Pearce and Cann, 1973) involving concentrations of $\mathrm{Ti}, \mathrm{Zr}, \mathrm{Sr}$, and $\mathrm{Y}$ in 109 samples with the composition of basalts and basic andesites $\left(45 \%-56 \% \mathrm{SiO}_{2}\right)$. Open squares indicate samples in the age range $27-29 \mathrm{Ma}$; several of these have characteristics of ocean-floor basalts or within-plate basalts. The hidden vertex is Zr. WPB = within-plate basalts; $\mathrm{LKT}=$ low-potassium tholeiitic basalts; $\mathrm{CAB}=$ calc-alkaline basalts; $\mathrm{IAB}=$ island-arc basalts; and $\mathrm{MORB}=$ mid-ocean-ridge (ocean-floor) basalts.

content; and lack of biogenic $\mathrm{CaCO}_{3}$. Volatile-free ICP-MS data are presented in Table 4.

Selected trace elements in the 16 samples that were analyzed by ICP-MS are plotted by age in Figure 14. The six age groups have significance because they correspond to (1) gaps in sampling, (2) epoch boundaries, or (3) changes in REE patterns (Fig. 15). The time intervals are $0-1,4-5,5-20,23.5-27.5(23.5 \mathrm{Ma}=$ Miocene/Oligocene boundary), 27.5-29, and 29-31 Ma. The only reason for subdivision of the interval $27.5-31 \mathrm{Ma}$ (6 samples) is to avoid cluttered graphs. All the volcanic detritus is enriched, relative to average
N-MORB, by about $10-30$ times in $\mathrm{Ba}, \mathrm{Rb}$, and $\mathrm{K}$, and by a factor of about 2 in $\mathrm{Sr}$. The concentrations of $\mathrm{Na}, \mathrm{P}, \mathrm{Zr}, \mathrm{Ti}, \mathrm{Y}$, and $\mathrm{V}$ are similar to, or slightly less than, their abundances in average N-MORB. Samples in the age range 27.5-29 Ma are the least fractionated. $\mathrm{Nb}$ concentrations are almost all less than $1.0 \mathrm{ppm}$ (Table 4). As a result, the ratio $\mathrm{Zr} / \mathrm{Nb}$ is well over 40 , in the range characteristic of average N-MORB and low-K tholeiites.

REE abundances (Table 4), normalized to primitive mantle, are presented for each of the age groups in Figure 15. Selected trace elements (Th, $\mathrm{Nb}, \mathrm{Zr}, \mathrm{Ti}, \mathrm{Y}$ ) are plotted adjacent to REEs of similar

Table 4. Volatile-free ICP-MS data, including rare-earth elements.

\begin{tabular}{|c|c|c|c|c|c|c|c|c|c|c|c|c|c|c|c|c|c|c|}
\hline \multirow{2}{*}{$\begin{array}{l}\text { Core, section, Depth } \\
\text { interval (cm) }\end{array}$} & \multirow{2}{*}{$\begin{array}{c}\text { Age } \\
\text { (mbsf) }\end{array}$} & \multirow[b]{2}{*}{ (Ma) } & \multicolumn{15}{|c|}{ Trace elements (ppm) } & \multirow[b]{2}{*}{$\mathrm{Sm}$} \\
\hline & & & Sc & $\mathrm{Rb}$ & $\mathrm{Sr}$ & $\mathrm{Y}$ & $\mathrm{Zr}$ & $\mathrm{Nb}$ & $\mathrm{Ba}$ & $\mathrm{Pb}$ & Th & $\mathrm{U}$ & $\mathrm{Li}$ & $\mathrm{La}$ & $\mathrm{Ce}$ & $\operatorname{Pr}$ & $\mathrm{Nd}$ & \\
\hline $126-790 \mathrm{~B}-8 \mathrm{H}-5,118-121$ & 68.78 & 0.07 & 33.34 & 6.10 & 210.37 & 24.87 & 63.60 & 1.23 & 82.57 & 4.17 & 0.44 & 0.20 & 6.22 & 4.20 & 11.14 & 1.87 & 9.72 & 2.87 \\
\hline 126-791A-28X-CC, $8-11$ & 247.03 & 0.12 & 28.66 & 5.28 & 215.13 & 24.50 & 55.48 & 0.81 & 253.49 & 95.41 & 0.35 & 0.28 & 6.16 & 3.52 & 9.39 & 1.57 & 8.62 & 2.75 \\
\hline 126-788D-7R-CC, $7-10$ & 279.48 & 4.17 & 41.23 & 12.25 & 162.96 & 20.85 & 44.85 & 0.51 & 59.55 & 3.06 & 0.18 & 0.20 & 13.49 & 2.11 & 6.04 & 1.11 & 6.02 & 2.15 \\
\hline $126-788 \mathrm{D}-9 \mathrm{R}-1,101-105$ & 297.91 & 4.23 & 39.35 & 10.95 & 164.11 & 20.92 & 49.08 & 0.47 & 52.28 & 2.24 & 0.14 & 0.14 & 17.45 & 2.02 & 5.91 & 1.07 & 6.15 & 2.24 \\
\hline $126-792$ E- 9 R- $3,68-73$ & 216.28 & 6.60 & 45.62 & 4.53 & 170.93 & 25.71 & 45.50 & 0.35 & 95.02 & 2.23 & 0.28 & 0.17 & 8.13 & 1.78 & 5.57 & 1.09 & 6.18 & 2.58 \\
\hline $126-792 \mathrm{E}-11 \mathrm{R}-1,87-90$ & 232.77 & 6.90 & 41.24 & 7.06 & 161.59 & 19.83 & 36.11 & 0.26 & 58.78 & 2.70 & 0.29 & 0.09 & 10.45 & 1.41 & 4.60 & 0.85 & 4.94 & 1.87 \\
\hline $126-793 \mathrm{~B}-3 \mathrm{R}-1,66-70$ & 604.96 & 15.10 & 45.64 & 12.36 & 168.31 & 22.56 & 39.66 & 0.64 & 241.83 & 5.42 & 0.88 & 0.18 & 19.54 & 2.74 & 7.43 & 1.24 & 6.54 & 2.37 \\
\hline $126-787 \mathrm{~B}-15 \mathrm{R}-4,26-29$ & 131.91 & 27.18 & 38.58 & 18.75 & 157.72 & 15.82 & 67.07 & 0.93 & 192.48 & 5.55 & 0.62 & 0.23 & 12.65 & 2.37 & 6.87 & 1.15 & 5.57 & 2.09 \\
\hline 126-792E-33R-1, $105-108$ & 445.25 & 27.40 & 37.40 & 21.06 & 132.02 & 12.07 & 56.29 & 0.76 & 471.97 & 4.23 & 0.97 & 0.56 & 9.52 & 1.74 & 4.58 & 0.79 & 3.74 & 1.36 \\
\hline $126-787 \mathrm{~B}-21 \mathrm{R}-1,65-69$ & 185.75 & 27.87 & 37.35 & 4.63 & 302.43 & 14.08 & 46.79 & 0.59 & 44.58 & 2.46 & 0.62 & 0.14 & 7.29 & 2.46 & 6.52 & 1.10 & 5.49 & 1.88 \\
\hline $126-787 \mathrm{~B}-23 \mathrm{R}-4,18-21$ & 209.08 & 28.00 & 31.15 & 5.61 & 267.42 & 16.36 & 50.22 & 0.72 & 49.43 & 2.10 & 0.35 & 0.14 & 9.64 & 3.24 & 8.54 & 1.42 & 7.41 & 2.23 \\
\hline $126-792 \mathrm{E}-47 \mathrm{R}-2,88-93$ & 581.51 & 28.67 & 39.07 & 8.30 & 110.62 & 19.79 & 47.94 & 0.65 & 50.04 & 2.05 & 0.31 & 0.18 & 12.44 & 3.43 & 8.21 & 1.36 & 6.90 & 2.16 \\
\hline $126-792 \mathrm{E}-56 \mathrm{R}-1,36-39$ & 665.76 & 28.92 & 39.81 & 2.14 & 231.20 & 16.45 & 42.64 & 0.52 & 26.35 & 1.60 & 0.26 & 0.11 & 11.81 & 2.94 & 7.43 & 1.25 & 6.31 & 1.87 \\
\hline 126-793B-51R-5, 6-9 & 1071.70 & 29.60 & 43.83 & 20.65 & 215.81 & 15.43 & 34.68 & 0.43 & 129.00 & 2.71 & 0.28 & 0.13 & 14.86 & 2.32 & 5.85 & 1.00 & 5.09 & 1.68 \\
\hline $793 \mathrm{~B}-68 \mathrm{R}-3,92-96$ & 1234.32 & 30.08 & 26.69 & 10.95 & 217.19 & 17.37 & 56.95 & 0.59 & 141.31 & 2.67 & 0.48 & 0.24 & 5.77 & 4.72 & 9.56 & 1.47 & 7.19 & 2.00 \\
\hline $126-792 \mathrm{E}-65 \mathrm{R}-1,111-116$ & 752.91 & 30.10 & 25.01 & 20.49 & 210.89 & 17.76 & 46.91 & 0.77 & 78.05 & 1.51 & 2.48 & 0.14 & 27.71 & 4.44 & 10.67 & 1.91 & 8.78 & 2.68 \\
\hline
\end{tabular}




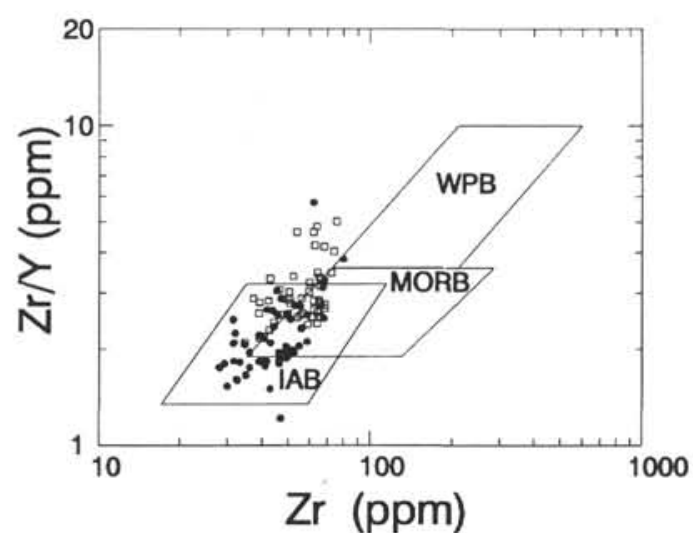

Figure 11. Petrological discrimination diagram (Pearce and Norry, 1979) involving concentrations of $\mathrm{Zr}$ and $\mathrm{Y}$ in 109 samples with the composition of basalts and basic andesites $\left(45 \%-56 \% \mathrm{SiO}_{2}\right)$. Open squares indicate samples in the age range $27-29 \mathrm{Ma}$; several of these plot outside all fields. $\mathrm{WPB}=$ within-plate basalts; $\mathrm{MORB}=$ mid-ocean-ridge basalts; and $\mathrm{IAB}=$ island-arc basalts. geochemical behavior. REE abundances are remarkably similar to those for average N-MORB (Fig. 15B). Relative Nb depletion, however, identifies the source materials as typical island-arc tholeiites. Variable $\mathrm{Th} / \mathrm{Nb}$ ratios are thought to reflect fluctuations in the relative contribution of elements from dehydration of the downgoing slab (e.g., Th) and from the supra-subduction zone mantle wedge (e.g., $\mathrm{Nb}$ ) (Briqueau et al., 1984).

A progressive change from slight LREE enrichment relative to chondritic mantle is present at $29-31 \mathrm{Ma}$, to flat patterns at $27.5-$ $29 \mathrm{Ma}$, followed by a fairly abrupt change to LREE depletion in samples younger than $27.5 \mathrm{Ma}$. This is accompanied by a crude decrease in $\mathrm{Th} / \mathrm{Nb}$ ratio. Similar variable LREE contents in a much smaller number of volcaniclastic samples from intraoceanic forearcs were reported by McLennan et al. (1990).

The history of LREE depletion is shown by a plot of $(\mathrm{La} / \mathrm{Yb})_{\mathrm{N}}$ vs. sample age (Fig. 16). The two samples younger than $1 \mathrm{Ma}$ $(0.07$ and $0.12 \mathrm{Ma})$ are both from the Sumisu Rift, a young backarc basin. They have $\mathrm{a}(\mathrm{La} / \mathrm{Yb})_{\mathrm{N}}$ ratio of about 1.0 , twice that for samples of age 4-27.7 Ma, and similar to that for samples in the age range 27.5-29 Ma that are more primitive than average. Young rift-facies basalts and rhyolites in Sumisu Rift also have a $(\mathrm{La} / \mathrm{Yb})_{\mathrm{N}}$ ratio of 1.0 or greater (Hochstaedter et al., 1990b; Gill et al., this volume).

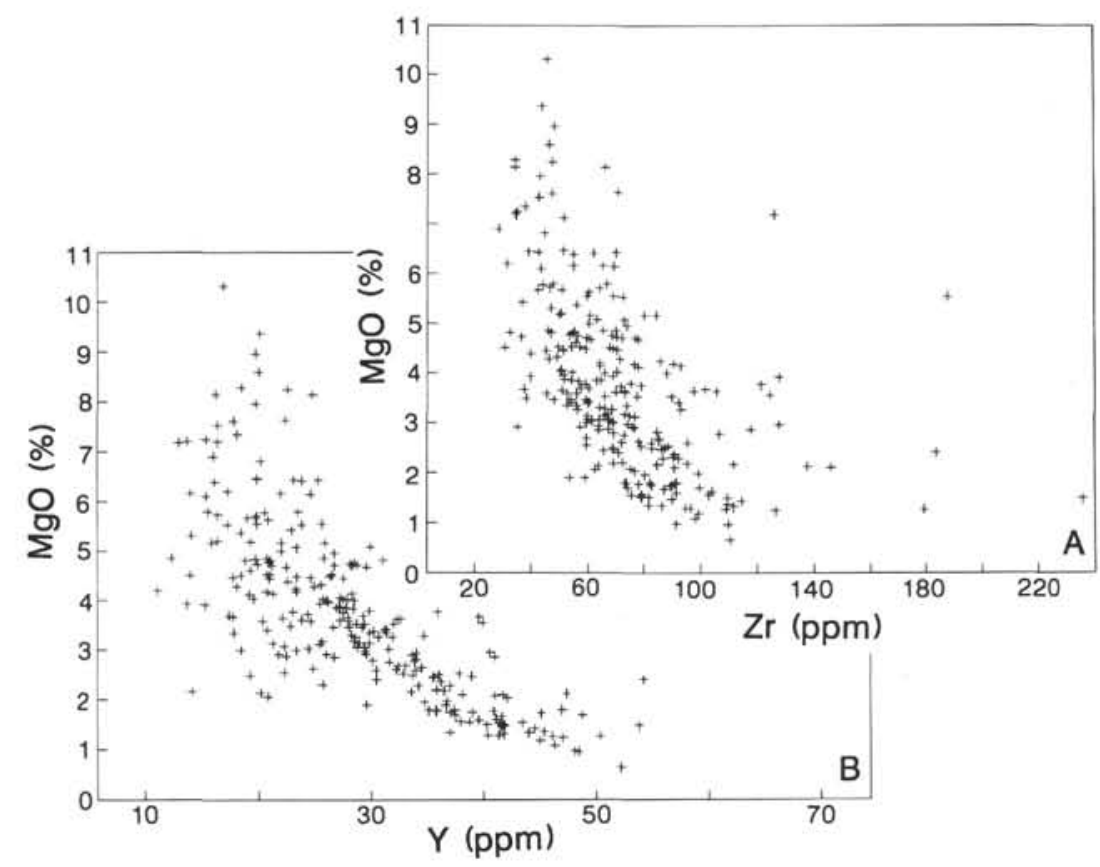

Figure 12. Relationships between contents of $\mathrm{MgO}, \mathrm{Zr}$, and $\mathrm{Y}$. Data for all samples are plotted.

Table 4 (continued).

\begin{tabular}{ccccccccccc}
\hline \multicolumn{10}{c}{ Trace elements (ppm) } \\
\hline Eu & Gd & Tb & Dy & Ho & Er & Tm & Yb & Lu & Hf & Ta \\
\hline 1.00 & 4.05 & 0.64 & 4.29 & 0.92 & 2.81 & 0.42 & 2.74 & 0.41 & 1.79 & 0.08 \\
1.03 & 3.83 & 0.63 & 4.15 & 0.90 & 2.87 & 0.44 & 2.69 & 0.42 & 1.75 & 0.08 \\
0.81 & 2.99 & 0.56 & 3.74 & 0.81 & 2.52 & 0.38 & 2.56 & 0.37 & 1.31 & 0.19 \\
0.78 & 3.01 & 0.55 & 3.76 & 0.83 & 2.56 & 0.40 & 2.48 & 0.40 & 1.68 & 0.04 \\
0.96 & 3.69 & 0.66 & 4.52 & 0.99 & 2.87 & 0.45 & 2.87 & 0.47 & 1.68 & 0.04 \\
0.71 & 2.89 & 0.50 & 3.49 & 0.76 & 2.37 & 0.35 & 2.31 & 0.38 & 1.26 & 0.03 \\
0.83 & 3.17 & 0.61 & 4.07 & 0.86 & 2.62 & 0.39 & 2.72 & 0.40 & 1.35 & 0.05 \\
0.78 & 2.63 & 0.50 & 3.85 & 0.81 & 2.60 & 0.40 & 2.73 & 0.36 & 2.08 & 0.07 \\
0.53 & 1.81 & 0.35 & 2.72 & 0.63 & 2.10 & 0.34 & 2.36 & 0.34 & 1.80 & 0.05 \\
0.66 & 2.29 & 0.43 & 2.66 & 0.58 & 1.81 & 0.27 & 1.73 & 0.28 & 1.46 & 0.11 \\
0.81 & 2.82 & 0.49 & 3.23 & 0.69 & 2.03 & 0.32 & 2.04 & 0.30 & 1.41 & 0.07 \\
0.75 & 3.02 & 0.51 & 3.45 & 0.77 & 2.22 & 0.32 & 2.05 & 0.35 & 1.47 & 0.05 \\
0.68 & 2.65 & 0.43 & 2.84 & 0.62 & 1.85 & 0.28 & 1.85 & 0.30 & 1.27 & 0.05 \\
0.65 & 2.45 & 0.40 & 2.70 & 0.56 & 1.87 & 0.27 & 1.80 & 0.27 & 1.04 & 0.04 \\
0.72 & 2.62 & 0.43 & 3.01 & 0.65 & 1.95 & 0.31 & 1.95 & 0.32 & 1.66 & 0.05 \\
1.05 & 3.23 & 0.54 & 3.28 & 0.77 & 2.22 & 0.38 & 2.05 & 0.38 & 1.42 & 0.11 \\
\hline
\end{tabular}

Pearce (1982) proposed plots of REE ratios to discriminate between magma series and to assess the extent and direction of subduction-related variations in REE abundances. A plot of $\mathrm{Ce} / \mathrm{Yb}$ vs. $\mathrm{Ta} / \mathrm{Yb}$ (Fig. 17) is consistent with the observation that the samples selected for ICP-MS analysis consist of arc tholeiitic detritus. The source rocks were apparently derived by partial melting of a mantle source that was depleted in LREE before its involvement in subduction-related processes (Pearce, 1982), like lavas of the Tonga Arc.

\section{ARC EVOLUTION}

As an example of the potential of sedimentary geochemistry in constraining the igneous geochemical evolution of ocean-island arcs, the Pliocene history at Site 788 was evaluated in some detail. These 


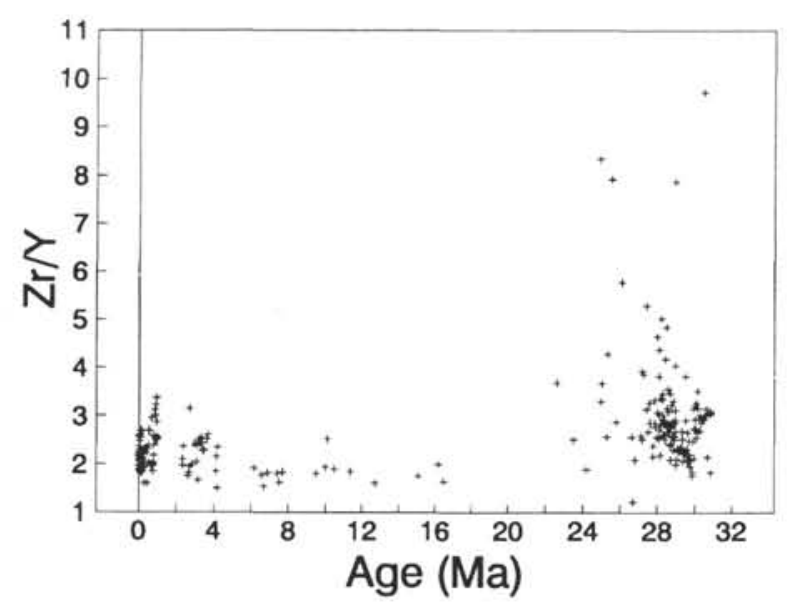

Figure 13. $\mathrm{Zr} / \mathrm{Y}$ vs. age. Age is taken from age-depth plots in Taylor, Fujioka, et al. (1990).
Table 5. Comparison of a Site 788 sand with igneous clasts.

\begin{tabular}{|c|c|c|c|}
\hline Sample & $126-788 \mathrm{C}-2 \mathrm{H}-1$ & $126-788 \mathrm{C}-2 \mathrm{H}-1$ & $126-788 \mathrm{C}-2 \mathrm{H}-1$ \\
\hline Interval (cm) & $6-10$ & $30-90$ & $36-38$ \\
\hline Material & Sand & Pumice & Shards \\
\hline \multicolumn{4}{|c|}{ Major elements (weight \%): } \\
\hline $\mathrm{SiO}_{2}$ & 66.94 & 67.15 & 68.57 \\
\hline $\mathrm{TiO}_{2}$ & 0.79 & 0.80 & 0.76 \\
\hline $\mathrm{Al}_{2} \mathrm{O}_{3}$ & 14.08 & 13.93 & 14.16 \\
\hline $\mathrm{Fe}_{2} \mathrm{O}_{3}$ & 5.70 & 6.99 & 6.93 \\
\hline $\mathrm{MgO}$ & 1.50 & 1.92 & 1.02 \\
\hline $\mathrm{CaO}$ & 4.42 & 4.91 & 4.79 \\
\hline $\mathrm{Na}_{2} \mathrm{O}$ & 4.01 & 3.40 & 3.65 \\
\hline $\mathrm{K}_{2} \mathrm{O}$ & 0.63 & 0.75 & 0.46 \\
\hline $\mathrm{P}_{2} \mathrm{O}_{3}$ & 0.13 & 0.14 & - \\
\hline \multicolumn{4}{|c|}{ Trace elements (ppm): } \\
\hline $\mathrm{Ba}$ & 274 & 232 & - \\
\hline $\mathrm{Sr}$ & 211 & 172 & - \\
\hline $\mathrm{Zr}$ & 79 & 72 & - \\
\hline $\mathrm{Nb}$ & 2 & 1 & - \\
\hline $\mathrm{Y}$ & 40 & 34 & - \\
\hline $\mathrm{Ni}$ & $<5$ & 8 & - \\
\hline Sc & 20 & 22 & - \\
\hline Reference & (1) & (2) & (3) \\
\hline
\end{tabular}

Notes: References are as follows: (1) this paper, (2) Gill et al. (this volume); and (3) Rodolfo et al. (this volume). Dashes = not determinable by electron microprobe.
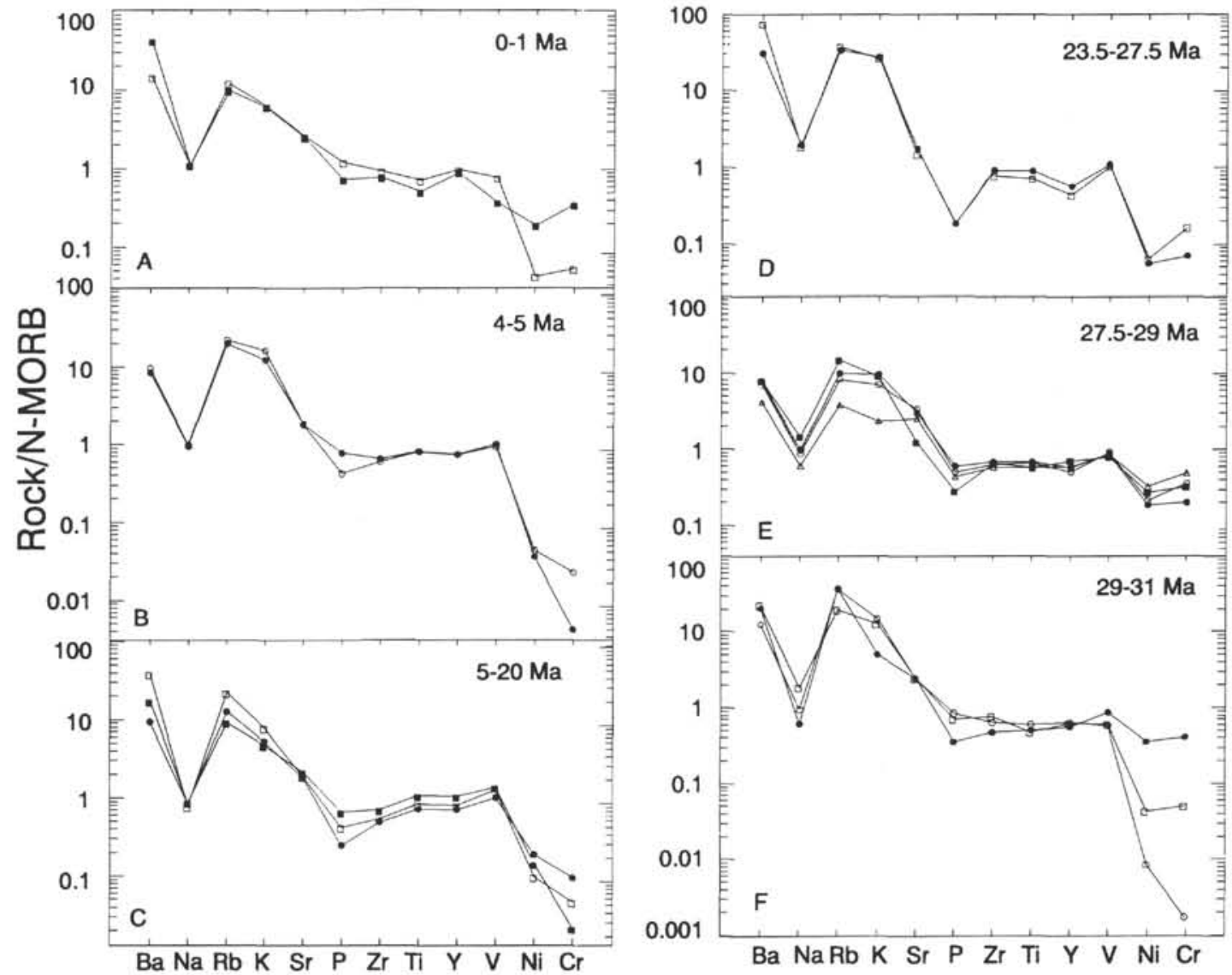

Figure 14. Variations in trace elements determined by AA (Na, K, P), XRF (Ti, V, Ni, Cr) and ICP-MS (Ba, Rb, Sr, Zr, Y). All element concentrations are normalized to those in average N-MORB (Table 1). Sample numbers, from highest to lowest Ba content for each time slice, are A = 0-1 Ma, Samples 126-791A-28X-CC, 8-11 cm, and 126-790B-8H-5, 118-121 cm; B = 4-5 Ma, Samples 126-788D-7R-CC, 7-10 cm, and -9R-1, 101-105 cm; C = 5-20 Ma, Samples 126-793B-3R-1, 66-70 cm, 126-792E$11 \mathrm{R}-1,87-90 \mathrm{~cm}$, and 126-792E-9R-3, 68-73 cm; D = 23.5-27.5 Ma, Samples 126-792E-33R-1, 105-108 cm, and 126-787B$15 R-4,26-29 \mathrm{~cm} ; \mathrm{E}=27.5-29 \mathrm{Ma}$, Samples 126-792E-47R-2, 88-93 cm, 126-787B-23R-4, 18-21 cm, 126-787B-21R-1, 65- $69 \mathrm{~cm}$, and 126-792E-56R-1, 36-39 cm; and F = 29-31 Ma, Samples 126-793B-68R-3, 92-96 cm, 126-793B-51R-5, 6-9 cm, and $126-792 \mathrm{E}-65 \mathrm{R}-1,111-116 \mathrm{~cm}$. 


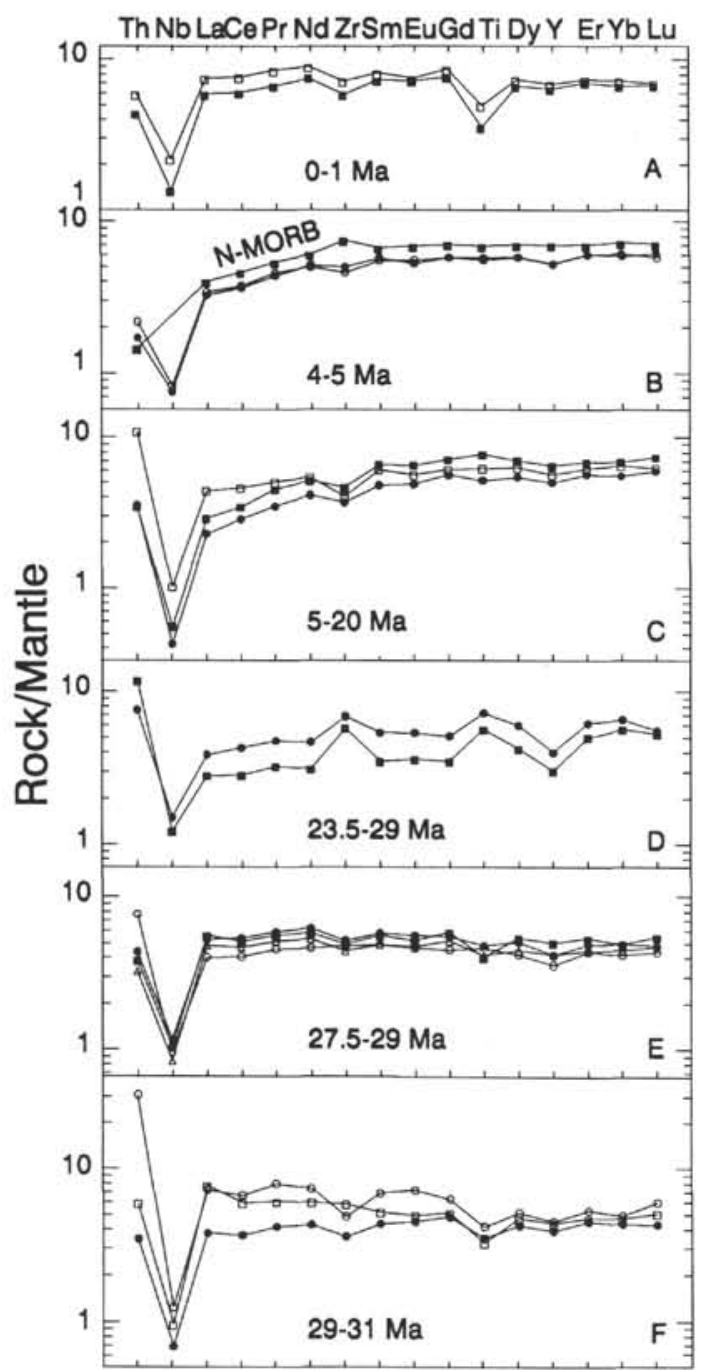

Th Nb LaCePrNdZrSmEuGdTi DyY Er Yb Lu

Figure 15. Variations in REE and selected trace elements determined by ICP-MS. All element concentrations are normalized to primitive mantle (Table 1). Sample numbers, from highest to lowest Th content for each time slice, are A = 0-1 Ma, Samples 126-790B-8H-5, 118-121 cm, and 126-791A-28X-CC, 8-11 cm; B = 4-5 Ma, Samples 126-788D-7R-CC, 7-10 cm, and -9R-1, 101$105 \mathrm{~cm} ; \mathrm{C}=5-20 \mathrm{Ma}$, Samples 126-793B-3R-1, 66-70 cm, 126-792E-11R-1, $87-90 \mathrm{~cm}$, and 126-792E-9R-3, 68-73 cm; D = 23.5-27.5 Ma, Samples 126-792E-33R-1, 105-108 cm, and 126-787B-15R-4, 26-29 cm; E = 27.5$29 \mathrm{Ma}$, Samples 126-787B-21R-1, 65-69 cm, 126-787B-23R-4, 18-21 cm, 126-792E-47R-2, 88-93 cm, and 126-792E-56R-1, 36-39 cm; and $\mathrm{F}=29$ $31 \mathrm{Ma}$, Samples 126-792E-65R-1, 111-116 cm, 126-793B-68R-3, 92-96 cm, and $126-793 \mathrm{~B}-51 \mathrm{R}-5,6-9 \mathrm{~cm}$.

sands were selected (1) because they are the most proximal to modern arc volcanoes and, therefore, most likely to reflect igneous compositions; and (2) because comparable chemical data at this site is known for individual pumice clasts and glass shards. Chronological data used in this section come from Taylor, Fujioka, et al. (1990) and Nishimura et al. (1991).

Table 5 compares analyses of a sand sample, a pumice clast, and glass shards from the same stratigraphic level near the top of the section at Site 788; the sand sample is essentially identical in composition to the average of all sands at this site younger than $3.15 \mathrm{Ma}$. The data show that the sands truly reflect the compositions of pieces of the igneous source rocks. Consideration of all available chemical data,

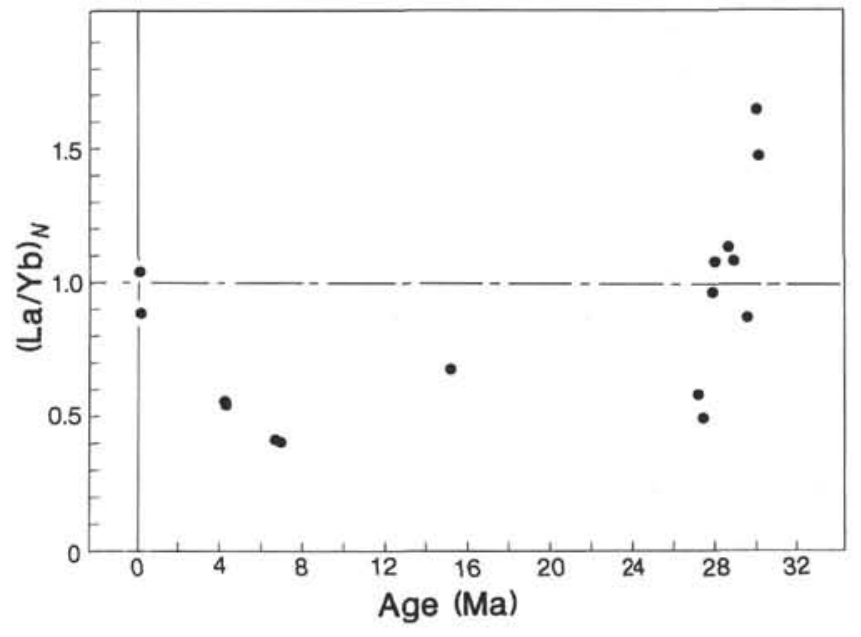

Figure 16. $(\mathrm{La} / \mathrm{Yb})_{\mathrm{N}}$ vs. age, in which concentrations are normalized to primitive mantle abundances (Table 1).

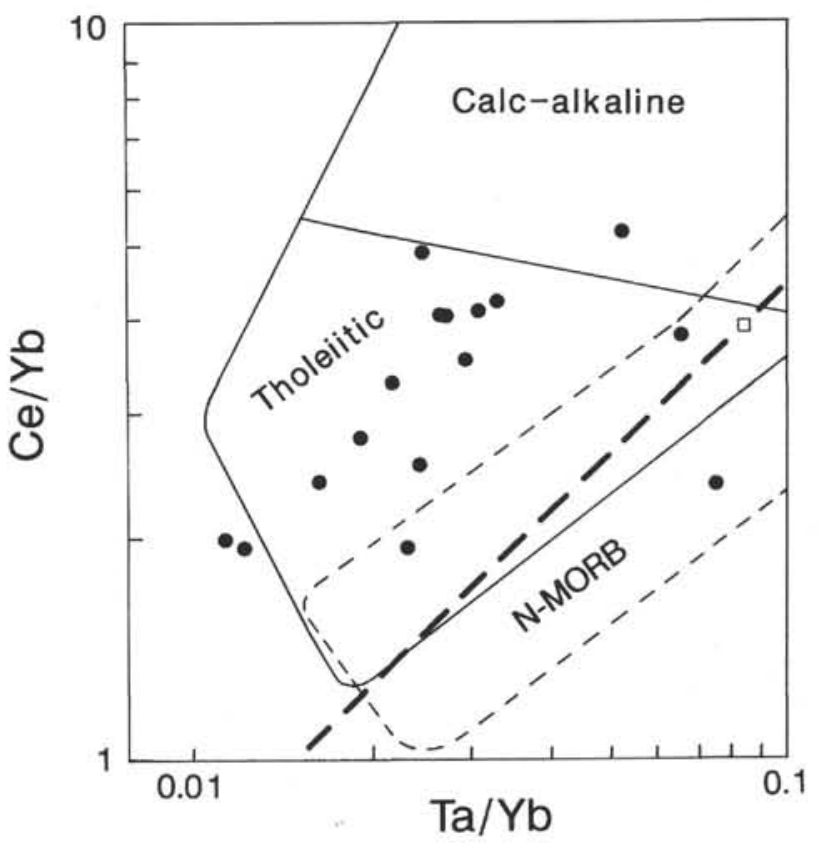

Figure 17. $\mathrm{Ce} / \mathrm{Yb}$ vs. $\mathrm{Ta} / \mathrm{Yb}$, with logarithmic scales. The fields of Pearce (1982) are indicated. The open square corresponds to the element ratios in primitive mantle (Table 1). Subduction-related variations tend to draw points vertically upward away from the bold dashed line through the primitive mantle composition (Pearce, 1982).

however, leads to the more general conclusion that the bulk sands are less extreme in composition than many individual pumice or lithic clasts (i.e., the sands are more intermediate and less basaltic or rhyolitic) and are less rhyolitic than many individual ash beds (Egeberg et al., this volume; Rodolfo et al., this volume). Many ash beds contain multiple populations of glasses (Rodolfo et al., this volume), of which the sands are approximately a weighted average, except that the sands are higher in $\mathrm{Na}$ and $\mathrm{K}$ and lower in $\mathrm{Ca}$. Microprobe data generally underestimate $\mathrm{Na}$ abundance, and the same is thought to be true here.

The variable sedimentation rates at Site 788 are thought to reflect the variable intensity of volcanic activity (Nishimura et al., 1991). The 
earliest interval of high sedimentation rates at 4.8-4.6 Ma was not sampled for this study. However, the next such interval at 4.2-4.1 Ma is marked by grayish black, basic, vitric sandstones (massive silicic eruptions were erroneously interpreted as an origin for these rocks in Taylor, Fujioka, et al., 1990, p. 418). The basic sandstones have low-alumina, iron-enriched, tholeiitic compositions $\left(\mathrm{SiO}_{2}=55 \%, \mathrm{Al}_{2} \mathrm{O}_{3}=14 \%\right.$ $15 \%, \mathrm{Fe}_{2} \mathrm{O}_{3}=12 \%-13 \%, \mathrm{MgO}=4.5 \%$, all on a volatile-free basis). Magmatic compositions of this kind imply plagioclase-dominated fractionation at low water pressure and oxygen fugacity. REE patterns for two sandstone samples (Fig. 15B) are depleted in LREEs, relative to which strong enrichments exist for $\mathrm{Pb}, \mathrm{Sr}, \mathrm{Ba}$, and, to a lesser extent, $\mathrm{K}$ and $\mathrm{Rb}$. Apart from the alkali enrichment, which could be attributed to alteration of the glass, these sandstones are almost indistinguishable in composition from the basic andesites of Tori Shima volcano, such as those from the 1939 eruption (Kuno, 1962).

After an apparent lull in arc activity, volcanism resumed at about $3.8 \mathrm{Ma}$ and continued until at least $2.35 \mathrm{Ma}$. The resulting sediments have been interpreted by Nishimura et al. (1991) as remobilized products of at least three major eruptive phases during 3.5-3.7 Ma (Sequence 1), 2.9-3.15 Ma (Sequence 2), and 2.35-2.7 Ma (Sequence 3). Each is a fines-depleted, upward-coarsening pumice deposit containing $<1 \%$ lithic clasts. The clasts originally settled through the water column, but the eruptions are thought to have been partly subaerial to explain the fines-depletion (Nishimura et al., 1991). Gill et al. (this volume) present analyses of individual pumice and lithic clasts, mostly from Sequence 2, and show that at least the oldest two sequences are heterolithic at two scales. Considering all particles, the lithics range in composition from basalt to andesite, whereas the pumices are silicic. In addition, the pumices are themselves variable, ranging from basic dacite to high-silica rhyolite; some single ash beds have a range of about $5 \% \mathrm{SiO}_{2}$.

The geochemical character of detritus from each of the three eruptive sequences is synthesized below, to illustrate how sand and sandstone geochemistry of volcaniclastic materials can be used to clarify the Pliocene-Pleistocene history of arc volcanism in this area. Subsequently, more general conclusions are drawn for pre-Pliocene history, which is less well constrained because (1) supporting data for pristine igneous particles are not available, and (2) the sandstones have been affected by diagenesis.

The Site 788 sandstones in Sequence 1, at 190-240 mbsf, have the composition of acid andesites $\left(62 \%-64 \% \mathrm{SiO}_{2}\right.$; Table 2$)$ in the lower $30 \mathrm{~m}$. Both the sandstones and the pumice clasts become more dacitic in the coarser top. The finer grained, overlying sandstones, interpreted as background sedimentation between large volcanic eruptions (Nishimura et al., 1991), revert to andesitic compositions.

In general, the sandstones in Sequence 2, at 100-140 mbsf, have more differentiated dacitic compositions but tend toward andesitic compositions at the top (Table 2). Pumices near the base of the sequence, from early in the eruptive cycle, are highly variable in composition, including acid andesite, low-silica rhyolite, and highsilica rhyolite. Pumice in Core $126-788 \mathrm{C}-12 \mathrm{H}-5$ is similar in composition to the most differentiated of the sandstones found in Cores $126-788 \mathrm{C}-13 \mathrm{H}$ to $-15 \mathrm{H}$. The pumice has a generally flat REE pattern and a clear, but somewhat muted, arc trace-element signature (Gill et al., this volume). Lithic clasts near the base of the sequence include acid andesite in Core 126-788C-14H-CC. At the very base, in Core $126-788 \mathrm{C}-15 \mathrm{H}-3$, a distinctive basalt clast is present that is similar in some respects to the backarc-basin basalts subsequently erupted in the Sumisu Rift (Gill et al., this volume). After this dacitic eruption, sandstones again reverted to andesitic compositions.

The sands of Sequence 3, at 35-75 mbsf, are dacitic. However, they differ from those of Sequence 2 by becoming more compositionally differentiated upsection. They also differ in trace element character. In particular, Sequence 3 sands have lower alkalis and $\mathrm{Zr}$, despite similar major element and $\mathrm{Y}$ contents. Their $\mathrm{Zr} / \mathrm{Y}$ ratios are $<2$, compared with values of $2.3-2.5$ in the older sediments of Sequence 2. It is noteworthy that this difference cannot be attributed to fractionation, unlike many other differences among the PliocenePleistocene dacitic-rhyolitic sands and sandstones.

The geochemical changes from Sequence 2 to Sequence 3 suggest a shift toward a more depleted source at 2.35-2.7 Ma. However, at least one pumice clast within Sequence 3 is similar in composition to rhyolite of the Sumisu Rift, rather than the Izu-Bonin Arc (Gill et al., this volume). Although no lithic clasts or sands in Sequence 3 are basaltic in composition, one glass shard with the composition of a Sumisu Rift basalt was found near the base of the sequence (compare Hochstaedter et al., 1990a, with Rodolfo et al., this volume).

A younger eruptive episode at Site 788, called Sequence 4, is late Pleistocene in age (Nishimura et al., 1991). Its sands and pumices continue the tendency toward depletion in the more incompatible trace elements. At Site 793, to the east of Site 788 (Fig. 1), coeval upper Pleistocene sediments are generally similar in composition to those in Sequence 4, being dacitic with low $\mathrm{K}$ and $\mathrm{Zr} / \mathrm{Y}$ of about 2.0. In contrast, to the north at forearc Sites 787 and 792, the detritus mostly has the composition of low-K basic andesite $(55 \%-60 \%$ $\mathrm{SiO}_{2}$ ). As in arcs, these more mafic materials have enrichments in alkali metals and alkaline earths, and low concentrations of $\mathrm{Ce}$ and high field strength elements (HFSE). The southern Sites 788 and 793 presumably are dominated by the predominantly silicic, late Pleistocene volcanic history of the Sumisu Jima and Minami Sumisu Jima calderas, whereas the more mafic materials at the northern Sites 787 and 792 probably reflect the volcanic products of Aoga Shima and the submarine caldera to its east.

In the backarc Sumisu Rift, late Pleistocene sands are also dacitic in average composition. A major contrast with older, pre-rift samples is the flat nature of the REE patterns obtained for two young samples of andesitic composition in the backarc (Fig. 15A), which have $(\mathrm{La} / \mathrm{Yb})_{\mathrm{N}}$ of about 1.0 , as opposed to ratios of about 0.5 before rifting (Fig. 16). These values are similar to those of rift-facies basalts on the floor of the basin (Hochstaedter et al., 1990b; Gill et al., this volume) and some pumice clasts in Sequence 2 (2.9-3.15 Ma), and are unlike ratios for lavas from the arc itself (Gill et al., this volume). For comparison, rift-facies rhyolites from the backarc basin area are enriched in LREEs, with $(\mathrm{La} / \mathrm{Yb})_{\mathrm{N}}$ of about 1.5.

The geochemical history of Pliocene-Pleistocene volcanism recorded in the sediments and sedimentary rocks can be interpreted in light of the inception of interarc rifting between 3.56 and $1.1 \mathrm{Ma}$ (Leg 126 Shipboard Drilling Party, 1989; Leg 126 Shipboard Scientific Party, 1989). Magma compositions for volcanoes at the volcanic front have had clear island-arc geochemical characteristics continuously from $4 \mathrm{Ma}$ to the present (Gill et al., this volume). Nevertheless, geochemically distinctive backarc-basin basalts and rhyolites appear as clasts and glass shards as early as about $3 \mathrm{Ma}$. The contribution of some detritus from LREE-enriched, rift-facies basalts is probably responsible for the flat REE patterns in two andesitic sand samples on the floor of the backarc basin. Volcanism in the south became more differentiated, from basalt to dacite, during a period of reduced volcanic output at $4 \mathrm{Ma}$, but it has remained predominantly daciticrhyolitic ever since. The predominance of silicic magmas in the arc since about $4.0 \mathrm{Ma}$ is thought to reflect a change to a more tensional stress regime during backarc rifting (Gill et al., this volume), facilitating upward movement of the relatively viscous silicic melts.

The composition of background sedimentation between large eruptive episodes is basic andesite in the north, east of Aoga Shima, and acid andesite to the south, between Sumisu Jima and Tori Shima. Thus, average volcanism is andesitic, even in this intraoceanic arc. However, pumice clasts and glass shards are more extremely basaltic and rhyolitic than are the sands and sandstones, so it remains uncertain whether the most common magmas were andesites or a bimodal assemblage of basalt and rhyolite. Petrographic analysis of the same sand and sandstone samples that were analyzed chemically indicates variable mixtures of clear, and dark brown to black, glass (Marsaglia, this volume), supporting the suggestion of bimodal magma compositions. 
The analysis we have presented for Pliocene-Pleistocene geochemistry can be extended to earlier time frames, but diagenesis of the volcaniclastic sandstones becomes increasingly significant with age (Marsaglia and Tazaki, this volume; Tazaki and Fyfe, this volume). Also, the sites, all in the forearc (Figs. 1 and 2), are more distant from the inferred positions of the contemporaneous arc volcanoes, which probably allowed greater mixing of components by transport processes.

During the Miocene, from about 15 to $5 \mathrm{Ma}$, volcanic activity in the Izu-Bonin Arc increased following the opening of the Shikoku Basin. The evidence for this volcanism is almost entirely from sediments at ODP Site 792 and DSDP Site 296 (Karig, Ingle, et al., 1975); no lavas of this age have been sampled along the Izu-Bonin Arc or Palau-Kyushu Ridge. Like the Pliocene record, most of the Miocene sandstones have the composition of low- $\mathrm{Al}$, low- $\mathrm{K}$ andesites. In general, the Miocene sandstones at Site 792 are more basic than the Pliocene ones at Site 788. They extend to greater depletion of LREEs (especially at $6 \mathrm{Ma}$ ), HFSEs, and alkalis. They also have higher $\mathrm{Ni}$ and $\mathrm{Cr}$ concentrations and, initially, higher $\mathrm{Ba}$ and $\mathrm{Th}$ contents (e.g., Core 126-793B-3R-1). Thus, magmas associated with this intensification of volcanism along the Izu-Bonin Arc in the Miocene were not shoshonitic (cf. Stern et al., 1988). However, if the higher Ba and Th contents are magmatic rather than diagenetic, then the revived volcanism, just as during the Pleistocene, had more arc tholeiitic traits (high $\mathrm{Ba} / \mathrm{Ce}$, low $\mathrm{Zr} / \mathrm{Y}$ and $[\mathrm{La} / \mathrm{Yb}]_{N}$ ratios) than did the 4-Ma basic andesites. That is, magmatism during the resumption of arc volcanism reflects the highest degrees of melting and the largest relative amounts of slab-derived components, whereas volcanism leading up to arc rifting (as well as the magmas erupted within the backarc rift itself) reflects lower percent fusion and a smaller recycled component.

Likewise, during 23.5-31 Ma, the sandstones were predominantly andesitic in composition, and the same distinction is preserved between more basic andesitic compositions $\left(50 \%-55 \% \mathrm{SiO}_{2}\right)$ in the north vs. more acid andesitic compositions $\left(55 \%-60 \% \mathrm{SiO}_{2}\right)$ in the south. These turbidite sandstones predate, and are partly contemporaneous with, the early stages of backarc spreading in the Shikoku Basin. They were coarsest in the time intervals 28-29 and 30-31 Ma, reflecting either bursts of volcanic activity or sea-level controls (Hiscott et al., this volume).

Drilling at Sites 792 and 793 penetrated volcanic basement to the forearc basin. At Site 793, the older turbidites (29-31 Ma) are slightly LREE-enriched, with $\mathrm{Zr} / \mathrm{Y}$ of about 3 (Fig. 13), $(\mathrm{La} / \mathrm{Yb})_{\mathrm{N}}=1-2$ (Fig. 16), $\mathrm{Y}=20-30 \mathrm{ppm}$, and $\mathrm{Ba}=100-200 \mathrm{ppm}$. These acid andesitic sandstones are interrupted twice by more basic, darker colored horizons. The first, at $1058-1154$ mbsf $(29.5-29.9 \mathrm{Ma})$, is the most basic package of sandstones encountered during Leg 126, with $\mathrm{MgO}=6 \%-8 \%, \mathrm{Ni}=30-60 \mathrm{ppm}, \mathrm{Cr}=40-160 \mathrm{ppm}$, and $\mathrm{Sc}=$ $30-40 \mathrm{ppm}$. It shows slight depletion in LREEs and is significantly more enriched in alteration-resistant incompatible trace elements than are the underlying lavas. The younger package of more basic sandstones, at 790-868 mbsf (25.75-28.2 Ma), is less rich in $\mathrm{Ni}$ and $\mathrm{Cr}$, with $\mathrm{MgO}=4.5 \%-8 \%$.

Most of the sandstones at the other two forearc sites (787 and 792) have the composition of basic andesites. None are as basic as the oldest dark-colored sandstone horizon at Site 793. Relative to differentiation indexes, the 26-29 Ma sandstones at Sites 787 and 792 have higher $\mathrm{TiO}_{2}, \mathrm{Y}$, and $\mathrm{Zr}$ contents than do the southern sandstones of similar age. This mimics the north-south contrasts between their underlying breccias and lavas (R. Taylor et al., this volume). Finally, the highly variable $\mathrm{P}_{2} \mathrm{O}_{5}$ contents observed in the lavas also characterize the sandstones at Site 792 , in which about $25 \%$ of the samples have $<0.03 \% \mathrm{P}_{2} \mathrm{O}_{5}$ (especially during $27.5-28.5 \mathrm{Ma}$ ), whereas the majority have $0.06 \%-0.12 \%$. In general, the REE patterns of the 27.5-29 Ma sandstones are flat, intermediate between the LREEdepleted, older basic sandstone package at Site 793, and the other two 29-31 Ma samples that are slightly enriched in LREEs (Fig. 15F). However, the youngest sandstones, deposited during 23.5-27.5 Ma, are consistently depleted in LREEs. These are the $\mathrm{P}_{2} \mathrm{O}_{5}$-poor sandstones noted above. The LREE depletion that appears first in these approximately 27.5-Ma sandstones has remained a characteristic of the main chain of the Izu-Bonin Arc to the present day; LREE depletion is only absent in very young andesitic samples from the backarc Sumisu Rift (Figs. 15 and 16); these may contain detritus from distinctive backarc volcanic rocks. [See note added in proof, following "Conclusions" section.]

Potential source areas for detritus in Oligocene samples are only known from the Bonin Islands (Kuroda and Shiraki, 1975; Umino, 1985 ) and from ODP Leg 125 sites on the outer-arc high (Fryer, Pearce, et al., 1990). The main volcanic source rocks through the early and late Oligocene, until about $27.5 \mathrm{Ma}$, were derived from a less depleted mantle source than were younger volcanic rocks. Although some samples have fairly high $\mathrm{MgO}$ contents, concentrations of $\mathrm{Y}$ and $\mathrm{Ti}$ are too high to infer a significant contribution from boninitic source rocks (Hickey and Frey, 1982; Rogers et al., 1989). Also, the shape of the REE plots is unlike the U-shaped plots that characterize some boninites (Murton, 1989).

A fundamental change in the trace element chemistry of volcaniclastic material occurred at about $27.5 \mathrm{Ma}$. In particular, $\mathrm{MgO}$ and $\mathrm{Cr}$ contents decrease and LREE depletion becomes a fixed characteristic of the sandstones for at least the following 25 m.y. (Fig. 16). The shift in geochemistry may reflect a change in provenance, perhaps from the region of the modern outer-arc high (e.g., Leg 125 forearc sites, Bonin Islands), east of the basin, to the position of the modern volcanic arc, west of the basin. Alternatively, there may have been an event that changed the composition of the mantle wedge beneath the arc, and thus the chemistry of derivative melts.

\section{CONCLUSIONS}

Turbidites from intraoceanic island arcs have generally igneous bulk major and trace element compositions that can be used to constrain the geochemical evolution of the arc. Conclusions are broadly similar to those derived by analysis of relict pyroxene crystals (Fujioka and Saito, this volume). The oldest sandstones, estimated to have an age of about $31 \mathrm{Ma}$, were derived from an arc tholeiitic, not boninitic, source. The 26-31 Ma sandstones farther to the north, at Sites 787 and 792, have higher relative concentrations of $\mathrm{Ti}, \mathrm{Zr}$, and $\mathrm{Y}$ than do those at southern Site 793.

In the Izu-Bonin Arc, magmas have become more LREE-depleted with time. A major change from flat or LREE-enriched patterns to LREE-depleted patterns occurred in the late Oligocene. This may correlate with a change from volcanic sources located trenchward of the modern forearc basin to sources in the vicinity of the modern volcanic arc.

Locally, in the backarc basin, sand samples that postdate eruption of rift-facies, LREE-enriched lavas have flat REE patterns that suggest mixing of some of this LREE-enriched material with detritus from the arc. Direct evidence for this addition comes from the presence of lithic clasts and glass shards of rift-facies basalt in sediments as old as $2.35-3.15 \mathrm{Ma}$.

Although average volcanism has remained andesitic in composition throughout the evolution of the Izu-Bonin Arc, it is unclear whether magmas were predominantly andesitic vs. a bimodal mixture of silicic and basic. Petrographic data (Marsaglia, this volume) are probably more useful for resolution of this question. Nevertheless, for more than 30 m.y., the average composition of volcaniclastic sediments and volcanism near Aoga Shima appears to have been more basic than to the south, near Sumisu Jima.

Although spatial variations in composition introduce uncertainty, it appears that volcanism became decidedly more silicic after a relative lull in activity at about $4 \mathrm{Ma}$. The increase in the proportion of silicic magmas is attributed to more easy ascent of these viscous melts because of recent tensional tectonics (Gill et al., this volume). 
[NOTE ADDED IN PROOF: New trace element data, acquired after acceptance of this paper, indicate that the transition to LREE depletion did not take place abruptly at $27.5 \mathrm{Ma}$, but instead took place sometime in the period 24-15 Ma, for which we currently have few ICP-MS analyses. Low values of $(\mathrm{La} / \mathrm{Yb})_{\mathrm{N}}$ in Samples $126-787 \mathrm{~B}-15 \mathrm{R}-4$, $26-29 \mathrm{~cm}$, and $126-792 \mathrm{E}-33 \mathrm{R}-1,105-108 \mathrm{~cm}$, may result from the unexpected diagenetic mobility of LREEs. These additional values are presented in Table 6.]

\section{Table 6. Additional values of $(\mathrm{La} / \mathrm{Yb})_{\mathrm{N}}$ determined by ICP-MS.}

\begin{tabular}{lcc}
\hline \multicolumn{1}{c}{$\begin{array}{c}\text { Core, section, } \\
\text { interval }(\mathrm{cm})\end{array}$} & $\begin{array}{c}\text { Age } \\
(\mathrm{Ma})\end{array}$ & $(\mathrm{La} / \mathrm{Yb})_{\mathrm{N}}$ \\
\hline 126-792E-23R-1, 118-121 & 12.70 & 0.33 \\
126-792E-28R-2, 94-97 & 22.60 & 0.98 \\
126-793B-20R-4, 91-95 & 24.95 & 2.54 \\
126-787B-14R-3, 72-76 & 26.80 & 1.58 \\
126-793B-26R-5, 125-129 & 27.80 & 2.25 \\
126-792E-42R-6, 80-83 & 28.45 & 1.11 \\
126-793B-36R-6, 103-107 & 28.85 & 1.35 \\
126-793B-54R-3, 103-107 & 29.69 & 0.79 \\
\hline
\end{tabular}

\section{ACKNOWLEDGMENTS}

Rock samples were crushed at the Geological Survey of Japan during the time that R. N. Hiscott held a Japanese Government Science and Technology Agency Fellowship. We thank the Department of Marine Geology, Geological Survey of Japan, for providing laboratory facilities and expertise. Rock analysis at Memorial University was expertly provided by Helen Gillespie (pellet preparation and data spreadsheets), Terry Wiseman (crushing), Gertrude Andrews (AA and $\mathrm{CaCO}_{3}$ ), Daryl Clarke (XRF), Simon Jackson (ICP-MS), and Henry Longerich (interference corrections). Elizabeth Hearn generated many of the graphs used in this paper and is thanked for providing several versions of some graphs to emphasize different features of the data. Preliminary advice to R. N. Hiscott on presentation and interpretation of data by Peter Cawood, George Jenner, and Nick Rogers is greatly appreciated.

\section{REFERENCES}

Bouma, A. H., 1962. Sedimentology of Some Flysch Deposits: Amsterdam (Elsevier).

Briqueau, L., Bougault, H., and Joron, J.-L., 1984. Quantification of Nb, Ta, $\mathrm{Ti}$ and $\mathrm{V}$ anomalies in magmas associated with subduction zones: petrographic implications. Earth Planet. Sci. Lett., 68:297-308.

Cas, R.A.F., and Wright, J. V., 1987. Volcanic Successions: Modern and Ancient: London (Allen and Unwin).

Egeberg, P., and Leg 126 Shipboard Scientific Party, 1990. Unusual composition of pore waters found in the Izu-Bonin fore-arc sedimentary basin. Nature, 344:215-218.

Fryer, P., Pearce, J. A., Stokking, L. B., et al., 1990. Proc. ODP, Init. Repts., 125: College Station, TX (Ocean Drilling Program).

Hickey, R. L., and Frey, F. A., 1982. Geochemical characteristics of boninite series volcanics: implications for their source. Geochim. Cosmochim. Acta, 46:2099-2115.

Hickey-Vargas, R. L., 1989. Boninites and tholeiites from DSDP Site 458, Mariana forearc. In Crawford, A. J. (Ed.), Boninites and Related Rocks: London (Unwin Hyman), 339-355.

Hochstaedter, A. G., Gill, J. B., Kusakabe, M., Newman, S., Pringle, M., Taylor, B., and Fryer, P., 1990a. Volcanism in the Sumisu rift. I. Major element, volatile and stable isotope geochemistry. Earth Planet. Sci. Lett., 100:179-194

Hochstaedter, A. G., Gill, J. B., and Morris, J. D., 1990b. Volcanism in the Sumisu Rift II. Subduction and non-subduction related components. Earth Planet. Sci. Lett., 100:195-209.
Hofmann, A. W., 1988. Chemical differentiation of the Earth: the relationship between mantle, continental crust, and oceanic crust. Earth Planet. Sci. Lett., 90:297-314.

Honza, E., and Tamaki, K., 1985. The Bonin Arc. In Naim, A.E.M., Stehli, F. G., and Uyeda, S. (Eds.), The Ocean Basins and Margins (Vol. 7): New York (Plenum), 459-502.

Jenner, G. A., Longerich, H. P., Jackson, S. E., and Fryer, B. J., 1990. ICP-MS: a powerful tool for high-precision trace element analysis in earth sciences: evidence from analysis of selected U.S.G.S. reference samples. Chem. Geol., 83:133-148.

Karig, D. E., Ingle, J. C., Jr., et al., 1975. Init. Repts. DSDP, 31: Washington (U.S. Govt. Printing Office).

Kobayashi, K., and Nakada, M., 1979. Magnetic anomalies and tectonic evolution of the Shikoku inter-arc basin. In Uyeda, S. et al. (Eds.), Geodynamics of the Western Pacific. Japan Sci. Soc. Press, Advances Earth Planet. Sci., 6:391-402.

Kuno, H., 1962. Catalogue of the Active Volcanoes of the World, Part II: Japan, Taiwan, and Marianas. Int. Assembly Volc. Chem. Earth's Interior.

Kuroda, N., and Shiraki, K., 1975. Boninite and related rocks of Chichi-Jima, Bonin Islands, Japan. Rep. Fac. Sci. Shizuoka Univ., 10:145-155.

Leg 126 Scientific Drilling Party, 1989. ODP Leg 126 drills the Izu-Bonin Arc. Geotimes, 34:36-38.

Leg 126 Shipboard Scientific Party, 1989. Arc volcanism and rifting. Nature, 342:18-20.

Matsuda, J., 1985. Sr isotopic studies of rocks from the Philippine Sea and some implications for the mantle material. In Shiki, T. (Ed.), Geology of the Northern Philippine Sea. Tokyo (Tokai Univ. Press), 63-78.

Mattey, D. P., Marsh, N. G., and Tarney, J., 1980. The geochemistry, mineralogy, and petrology of basalts from the West Philippine and Parece Vela basins and from the Palau-Kyushu and West Mariana ridges, Deep Sea Drilling Project Leg 59. In Kroenke, L., and Scott, R., et al., Init. Repts. DSDP, 59: Washington (U.S. Govt. Printing Office), 753-796.

McLennan, S. M., Taylor, S. R., McCulloch, M. T., and Maynard, J. B., 1990. Geochemical and Nd-Sr isotopic composition of deep-sea turbidites: crustal evolution and plate tectonic associations. Geochim. Cosmochim. Acta, 54:2015-2050.

Migdisov, A. A., Miklishansky, A. Z., Saveliev, B. V., Bredanova, N. V., Girin, Y. P., Pavlutshkaya, V. I., and Yakovlev, Y. V., 1981. Neutron activation analysis of rare earth elements and some other trace elements in volcanic ashes and pelagic clays, Deep Sea Drilling Project Leg 59. In Kroenke, L., Scott, R., et al., Init. Repts. DSDP, 59: Washington (U.S. Govt. Printing Office), 653-668.

Miyashiro, A., 1974. Volcanic rock series in island arcs and active continental margins. Am. J. Sci., 274:321-355.

Murton, B. J., 1989. Tectonic controls on boninite genesis. In Saunders, A. D. and Norry, M. J. (Eds.), Magmatism in the Ocean Basins. Geol. Soc. Spec. Publ. London, 42:347-377.

Nesbitt, H. W., and Young, G. M., 1989. Formation and diagenesis of weathering profiles. J. Geol., 97:129-147.

Nishimura A., Marsaglia, K. M., Rodolfo, K., Colella, A., Hiscott, R. N., Tazaki, K., Gill, J. B., Janecek, T., Firth, J., Isiminger-Kelso, M., Herman, Y., Taylor, R. N., Taylor, B., Fujioka K., and Leg 126 Scientific Party, 1991. Pliocene-Quaternary submarine pumice deposits in the Sumisu Rift area, Izu-Bonin Arc. In Smith, G., and Fiske, R. (Eds.), Sedimentation in Volcanic Settings. Spec. Publ., Soc. Econ. Paleontol. Mineral., 45:201-208.

Pearce, J. A., 1982. Trace element characteristics of lavas from destructive plate boundaries. In Thorpe, R. S. (Ed.), Andesites, Orogenic Andesites and Related Rocks: New York (Wiley), 525-549.

Pearce, J. A., and Cann, J. R., 1973. Tectonic setting of basic volcanic rocks determined using trace element analyses. Earth Planet. Sci. Lett., 19:290-300.

Pearce, J. A., and Norry, M. J., 1979. Petrogenetic implications of Ti, Zr, Y, and $\mathrm{Nb}$ variations in volcanic rocks. Contrib. Mineral. Petrol., 69:33-47.

Peccerillo, A., and Taylor, S. R., 1976. Geochemistry of Eocene calc-alkaline volcanic rocks from Kastamonu area, northern Turkey. Contrib. Mineral. Petrol., 58:63-81.

Rogers, N. W., MacLeod, C. J., and Murton, B. J., 1989. Petrogenesis of boninitic lavas from the Limassol Forest Complex, Cyprus. In Crawford, A. J. (Ed.), Boninites and Related Rocks: London (Unwin Hyman), 289-313.

Scott, R. B., 1980. Petrology and geochemistry of arc tholeiites on the Palau-Kyushu Ridge, Site 448, Deep Sea Drilling Project Leg 59. In Kroenke, L., Scott, R., et al., Init. Repts. DSDP, 59: Washington (U.S. Govt. Printing Office), 681-692. 
1983. Magmatic evolution of island arcs in the Philippine Sea. In Hilde, T.W.C., and Uyeda, S. (Eds.), Geodynamics of the Western PacificIndonesian Region. Am. Geophys. Union, Geol. Soc. Am., 173-187.

Shervais, J. W., 1982. Ti-V plots and the petrogenesis of modern and ophiolitic lavas. Earth Planet. Sci. Lett., 59:101-118.

Shiraki, K., 1978. Chromium: abundance in common igneous rocks. Handbook of Geochemistry, II/5: Berlin (Springer-Verlag), 24-E-1-24-E-8.

Stern, R. J., Bloomer, S. H., Lin, P.-N., Ito, E., and Morris, J., 1988. Shoshonitic magmas in nascent arcs: new evidence from submarine volcanoes in the northern Marianas. Geology, 16:426-430.

Sun, S.-S., and McDonough, W. F., 1989. Chemical and isotopic systematics of oceanic basalts: implications for mantle composition and processes. In Saunders, A. D., and Norry, M. J. (Eds.), Magmatism in the Ocean Basins. Geol. Soc. Spec. Publ. London, 42:313-345.

Taylor, B., Fujioka, K., et al., 1990. Proc. ODP, Init. Repts., 126: College Station, TX (Ocean Drilling Program).
Taylor, S. R., 1969. Trace element chemistry of andesites and associated calc-alkaline rocks. In McBirney, A. R. (Ed.), Proceedings of the Andesite Conference. Bull. Oregon Dept. Geol. Mineral. Industries, 65:43-64.

Taylor, S. R., and McLennan, S. M., 1985. The Continental Crust: Its Composition and Evolution: Oxford (Blackwell Scientific).

Tsunakawa, H., 1983. K-Ar dating on volcanic rocks in the Bonin Islands and its tectonic implication. Tectonophysics, 95:221-232.

Umino, S., 1985. Volcanic geology of Chichijima, the Bonin Islands (Ogasawara Islands). J. Geol. Soc. Jpn., 91:505-523.

Date of initial receipt: 13 December 1990

Date of acceptance: 9 September 1991

Ms 126B-150 